\title{
Equilibria and stability boundaries of shallow arches under static loading in a thermal environment
}

\author{
Behrang Moghaddasie ${ }^{\mathrm{a}}$, Ilinca Stanciulescu ${ }^{\mathrm{b}, *}$ \\ ${ }^{a}$ Department of Civil Engineering, Ferdowsi University of Mashhad, P.O. Box 91775-1111, \\ Mashhad, Iran \\ ${ }^{b}$ Department of Civil and Environmental Engineering, Rice University, Houston, TX \\ 77005, U.S.A
}

\begin{abstract}
The structural behaviour of shallow arches is complex and can be influenced by many parameters. In this paper, the response of a half-sine shallow arch under static loading in a thermal environment is investigated. The arch has pinned supports and the material behaviour is assumed elastic. The exact displacement field, load-bearing capacity and the locus of critical points are obtained. Boundaries of domains with different stability behaviour (e.g., different number of limit and bifurcation points) are also determined. Three types of loading (concentrated, uniform and asymmetrical uniform) are examined. The primary equilibrium paths are verified against results obtained from finite element simulations. The proposed method is robust and accurate.
\end{abstract}

Keywords: Shallow arch, Equilibrium path, Stability boundary, Critical load

\section{Introduction}

Shallow arches are widely used in civil, mechanical and aerospace engineering as stand-alone structures or components of more complex systems. Subsequently, the analysis of their behaviour has always been of interest to researchers and engineers. One type of analysis relies on the derivation of the exact solution based on some simplifying assumptions $[1,2]$. This is the approach that is also

\footnotetext{
*Corresponding author. Tel.: +1713 348 4704; fax: +1713 3485268.

Email address: ilinca@rice.edu (Ilinca Stanciulescu)
} 
taken in this paper. A different approach is usually based on non-linear finite element modelling $[3,4,5]$. A disadvantage of this procedure is that it requires the magnitude of independent parameters defined in the problem to be constant along the analysis. Hence, for each specific configuration a separate analysis is required.

A loss of stability that can frequently be observed in the response of shallow arches, is the snap-through. Snap-through is usually accompanied by large deformations and large stress reversals therefore accelerating fatigue. For these reasons, understanding snap-through and having the ability to estimate the associated stability boundaries are crucial in structural design $[6,7,8]$. The equilibrium state, displacement field, load-bearing capacity and snap-through of these systems are extensively discussed in the literature $[9,10,11]$. In most cases, it is assumed that the arch keeps its elastic characteristics throughout the analysis. Reported experimental results support this assumption [12, 13, 14]. The geometry of the arch is a design parameter that can influence the loadbearing capacity. Half-sine, circular and parabolic shapes have been studied $[15,16,17]$ and a variety of loading conditions have been investigated, such as concentrated loads $[18,19,20]$, distributed and end moments loads [21, 22, 23], and variations in the thermal fields or support conditions [5, 24, 25].

This paper introduces a semi-analytical procedure to find the exact solution to a half-sine pinned shallow arch under transversal loading. In addition, the effects of variation in initial temperature on the displacement field, equilibrium paths and locus of critical points are investigated. For this purpose, the governing equations of the arch are obtained based on non-dimensionalized parameters, and the displacement field is expanded into Fourier sine series. By equating the residual force to zero, primary and bifurcated equilibrium paths are derived. In order to find critical points (limit and bifurcation points), an extra constraint is added to the equilibrium equations. Furthermore, the natural frequencies of the arch in the vicinity of equilibrium states are calculated. The proposed method is applied to three different types of static loadings (concentrated, uniform and asymmetrical uniform). The superiority of the proposed method is shown in 
its ability to: (a) find the exact solution of the displacement field, equilibrium paths, locus of critical points and natural frequencies of the arch; (b) perform parametric analyses instead of multiple analyses with specified values; and (c) predict critical points without tracing the equilibrium path. The only disadvantages of the proposed method are the limitations coming from the simplifying assumptions used in the analysis.

The rest of the paper is organized as follows. In Section 2, the governing equations of the arch under arbitrary vertical loading and the corresponding equilibrium states are derived. In Section 3, the critical points (limit and bifurcation points) are defined and the critical state constraint is discussed. Section 4 introduces the evaluation of natural frequencies in the vicinity of equilibrium states. Section 5 presents the governing equations of a half-sine shallow arch, and the solution (with the proposed method) for the displacement field, the equilibrium paths and the locus of critical points for several loading patterns. Finally, concluding remarks are given in Section 6 .

\section{Governing equations}

In this section, the governing equations of a half-sine pin-ended shallow arch (Figure 1) under an arbitrary vertical loading $Q^{*}$ (a) are derived. A modified Bernoulli beam theory with large transversal displacements is used. The material is isotropic and homogeneous, and no transverse temperature gradient is considered. $E, \rho$ and $\alpha^{*}$ represent the Young's modulus, mass density and thermal expansion coefficient, respectively. $A$ is the area and $I$ denotes the moment of inertia of the cross section. All these parameters are assumed to be constant over the span $L$.

The assumptions used for the analysis are: (1) the axial force is constant over the span $[2,6,23]$; (2) the material is elastic; (3) out-of-plane deflections are neglected [13]; and (4) the range of displacements and curvatures of the arch is small in comparison with the length of the span $\left(0 \leq y_{\max } / L<1 / 10-1 / 50\right)$ 
(a)



(b)

$Q_{1}^{*}$

(c)

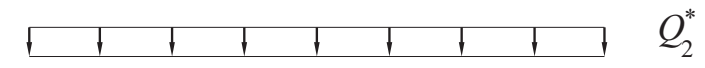

(d)
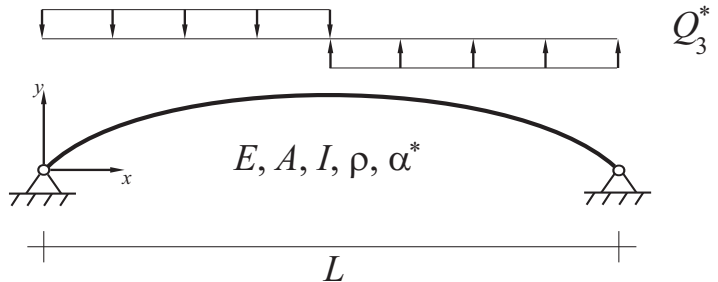

Figure 1: A half-sine pinned shallow arch under (a) arbitrary, (b) concentrated, (c) uniform and (d) asymmetric uniform loadings

[6]. The equation of motion can now be written as [17, 21, 26]:

$$
\rho A y_{, t t}=-E I\left(y-y_{0}\right)_{, x x x x}+P^{*} y_{, x x}-Q^{*},
$$

where, $y_{0}$ is the initial shape of the arch, the subscripts ", $t$ " and ", $x$ " show the partial differentiation with respect to the time and the longitudinal position, respectively, and $P^{*}$ represents the axial force:

$$
P^{*}=E A\left(-\alpha^{*} \Delta T_{0}+\frac{1}{2 L} \int_{0}^{L}\left(y_{, x}^{2}-y_{0, x}^{2}\right) d x\right),
$$

where $\Delta T_{0}$ denotes the initial temperature change before loading. In Eq. (2), since the supports are fixed in the direction of $x$, non-zero $\Delta T_{0}$ causes initial compressive or tensional axial thermal stress $\left(-E \alpha^{*} \Delta T_{0}\right)$. Eqs. (1) and (2) can be rewritten in dimensionless form:

$$
\begin{gathered}
u_{, \tau \tau}=-\left(u-u_{0}\right)_{, \xi \xi \xi \xi}+P u_{, \xi \xi}-c Q \\
P=-\alpha \Delta T_{0}+\frac{1}{2 \pi} \int_{0}^{\pi}\left(u_{, \xi}^{2}-u_{0, \xi}^{2}\right) d \xi
\end{gathered}
$$

where,

$$
\left(u, u_{0}\right)=\frac{1}{r}\left(y, y_{0}\right), \xi=\frac{\pi}{L} x, \tau=\frac{\pi^{2} r}{L^{2}} \sqrt{\frac{E}{\rho}} t
$$




$$
Q=\frac{L^{3}}{c \pi^{3} E I r} Q^{*}, \alpha=\frac{L^{2}}{\pi^{2} r^{2}} \alpha^{*} .
$$

The parameter $r$ is the radius of gyration of the cross section $(r=\sqrt{I / A})$. The arbitrary constant $c$ is just a scaling factor and has no effect on the final result. In this paper, for convenience, $c$ is assumed to be $\pi / 2$ and $\pi / 4$ for concentrated and distributed loading problems, respectively. Since a modified Bernoulli theory is applied, the shear modulus and Poisson's ratio do not appear in the formulation. The boundary conditions for Eq. (3) are

$$
u(0)-u_{0}(0)=u_{, \xi \xi}(0)-u_{0, \xi \xi}(0)=u(\pi)-u_{0}(\pi)=u_{, \xi \xi}(\pi)-u_{0, \xi \xi}(\pi)=0 .
$$

By considering the boundary conditions given in (6), the displacement field can be expanded into Fourier sine series. Consequently, the initial and deformed shapes of the shallow arch in the dimensionless configuration are

$$
\begin{gathered}
u_{0}(\xi)=h \sin \xi \\
u(\xi, \tau)=\sum_{n=1}^{\infty} \alpha_{n}(\tau) \sin n \xi .
\end{gathered}
$$

Here, $h$ is the dimensionless rise of the arch. The load $Q$ can be written via the Fourier series:

$$
Q=\frac{1}{c} \sum_{n=1}^{\infty} Q_{n} \sin n \xi
$$

where,

$$
Q_{n}=\frac{2 c}{\pi} \int_{0}^{\pi} Q \sin n \xi d \xi, n=1,2, \ldots
$$

By substituting Eqs. (7)-(9) into (3), and equating the coefficients of $\sin n \xi$ from both sides, the following set of equations is obtained:

$$
\ddot{\alpha}_{n}=-n^{4} \alpha_{n}-p n^{2} \alpha_{n}-q_{n}, n=1,2, \ldots
$$

In this equation, $q_{1}=Q_{1}-h$ and $q_{n}=Q_{n}$ for $n=2,3, \ldots$, and $p$ is

$$
p=-\alpha \Delta T_{0}-\frac{h^{2}}{4}+\sum_{k=1}^{\infty} \frac{k^{2} \alpha_{k}^{2}}{4}
$$

$(\bullet)$ denotes derivatives of $(\bullet)$ with respect to $\tau$. By solving the differential equations (11), one can calculate the variations in the mode coefficients $\alpha_{n}$, and subsequently, obtain the dynamic response of the shallow arch. 
At equilibrium, $\ddot{\alpha}_{n}=0$ for $n=1,2, \ldots$ and Eq. (11) becomes:

$$
R_{n}=0: n^{4} \alpha_{n}+p n^{2} \alpha_{n}+q_{n}=0, n=1,2, \ldots,
$$

where, $R_{n}$ is the residual (unbalanced) dimensionless force. The set of $\alpha_{n}$ satisfying Eq. (13), defines the equilibrium states that correspond to the given $Q$.

Note that the system is subjected to an initial temperature change $\Delta T_{0}$ but then the temperature remains constant during the static loading. As a result, material characteristics are constant while tracing the equilibrium path and finding critical points. For example, for a Young's modulus $E$ that is a function of $\Delta T_{0}\left(E=E\left(\Delta T_{0}\right)\right)$, first, the magnitude of $E$ is calculated based on the value of $\Delta T_{0}$; then, the static loading is applied, and the formulation given in this paper can be used to analyse the shallow arch.

\section{Critical points}

In this section, the critical states of the shallow arch are obtained. If $Q$ is a function of a load parameter $\lambda(Q=Q(\lambda))$, the solution to Eq. (13) describes a relationship between the displacements and the load parameter, i.e., the equilibrium path in the space of $(u, \lambda)$. Each point on this path is an equilibrium point. Figure 2 shows an example of an equilibrium path.



Figure 2: Primary (black) and secondary (gray) equilibrium paths 
The critical points are those points along the equilibrium path where sudden changes in the behaviour of the arch are observed. Two types of critical points are discussed in what follows: (1) limit points, where the variation of the load parameter $\lambda$ along the equilibrium path is equal to zero (points $\mathbf{A}$ in Figure 2), and (2) bifurcation points, located at the intersection of two or more equilibrium paths (points B in Figure 2). This figure is just a schematic example to show the possibility of the existence of limit and bifurcation points on the equilibrium path. There could exist more or fewer critical points, and either limit or bifurcation points can appear first on the path. These issues are examined later in the article.

The (modal) tangent stiffness matrix is defined as follows:

$$
K_{n m}=\frac{\partial R_{n}}{\partial \alpha_{m}}=\frac{n^{2} m^{2}}{2} \alpha_{n} \alpha_{m}+n^{2}\left(n^{2}+p\right) \delta_{n m}, n, m=1,2, \ldots
$$

Here, $\delta_{n m}$ is the Kronecker delta. The matrix is singular at the critical points:

$$
\left|K_{n m}\right|=\left(\prod_{k=1}^{\infty} \frac{k^{4}}{2}\right)\left|\alpha_{n} \alpha_{m}+\beta_{n m}\right|=0
$$

where,

$$
\beta_{n m}=\frac{n^{2}\left(n^{2}+p\right)}{n^{2} m^{2} / 2} \delta_{n m}, n, m=1,2, \ldots
$$

Eq. (15) can be rewritten in a simpler form:

$$
\prod_{k=1}^{\infty} \beta_{k k}+\sum_{n=1}^{\infty}\left(\alpha_{n}^{2} \prod_{\substack{k=1 \\ k \neq n}}^{\infty} \beta_{k k}\right)=0
$$

At critical points, Eq. (17) is always satisfied.

\section{Natural frequencies}

In this section, the calculation of natural frequencies in the vicinity of equilibrium points is described. While the paper does not introduce dynamic analyses, we introduce this section due to the similarities between the equations identifying the natural frequencies and other equations that are analytically solved 
here. These similarities allow for the calculation of the natural frequencies analytically, while a full transient analysis would require numerical integration.

A small perturbation factor $\varepsilon_{n}(\tau)$ is added to mode coefficients at the equilibrium state $\left(\alpha_{n}^{e}\right)$ [14]. The perturbed mode coefficients $\alpha_{n}(\tau)$ become

$$
\alpha_{n}(\tau)=\alpha_{n}^{e}+\varepsilon_{n}(\tau), n=1,2, \ldots
$$

By substituting Eq. (18) into (11) one can obtain a set of linear equations:

$$
\sum_{m=1}^{\infty}\left(M_{n m}^{e} \ddot{\varepsilon}_{n}+K_{n m}^{e} \varepsilon_{n}\right)=0, n=1,2, \ldots
$$

where, $M_{n m}^{e}$ and $K_{n m}^{e}$ are defined as

$$
\left\{\begin{array}{l}
M_{n m}^{e}=\delta_{n m} \\
K_{n m}^{e}=\frac{n^{2} m^{2}}{2} \alpha_{n}^{e} \alpha_{m}^{e}+n^{2}\left(n^{2}+p\right) \delta_{n m}
\end{array} \quad, n, m=1,2, \ldots\right.
$$

In order to find the natural frequencies of the shallow arch $(\omega)$, the following constraint is enforced:

$$
\left|K_{n m}^{e}-\omega^{2} M_{n m}^{e}\right|=\left(\prod_{k=1}^{\infty} \frac{k^{4}}{2}\right)\left|\alpha_{n}^{e} \alpha_{m}^{e}+\beta_{n m}^{\prime}\right|=0 .
$$

Eq. (22) shows the value of $\beta_{n m}^{\prime}$ :

$$
\beta_{n m}^{\prime}=\frac{n^{2}\left(n^{2}+p\right)-\omega^{2}}{n^{2} m^{2} / 2} \delta_{n m}, n, m=1,2, \ldots
$$

Similar to Eq. (17), the constraint (21) can be simplified:

$$
\prod_{k=1}^{\infty} \beta_{k k}^{\prime}+\sum_{n=1}^{\infty}\left(\left(\alpha_{n}^{e}\right)^{2} \prod_{\substack{k=1 \\ k \neq n}}^{\infty} \beta_{k k}^{\prime}\right)=0
$$

In the case of $\alpha_{n}^{e}=0$ for some $n$, the value of $\beta_{n n}^{\prime}$ is equal to zero (Eq. (23)). Consequently, from Eq. (22), the corresponding natural frequency $\omega_{n}=$ $n \sqrt{n^{2}+p}$ when $\alpha_{n}^{e}=0$. The authors' experience shows that for sufficiently large value of $n$, this equation can approximately obtain $\omega_{n}$ corresponding to $\alpha_{n}^{e} \neq 0$. A complex root for $\omega_{n}$ shows the instability of the equilibrium state in the direction of $\sin n \xi$. 


\section{Application to specific transversal loadings}

In this section, the behaviour of shallow arches under a variety of transversal loadings is investigated.

\subsection{Concentrated loading at the midpoint}

First, the case of the shallow arch under a concentrated load at the midpoint (Figure $1(\mathrm{~b})$ ) is analysed. For this purpose, the non-dimensionalized load $Q_{1}$ can be expressed as a function of the load parameter $\lambda$ :

$$
Q_{1}=\lambda \delta(\xi-\pi / 2)
$$

where, $\delta$ denotes the Dirac delta function. For convenience, the scaling factor $c$ is assumed to be equal to $\pi / 2$ in (10). As a result, the value of $q_{n}$ for $n=1,2, \ldots$ becomes

$$
\begin{cases}q_{1}=\lambda-h & \\ q_{2 i+1}=(-1)^{i} \lambda & , i=1,2, \ldots \\ q_{2 i}=0 & , i=1,2, \ldots\end{cases}
$$

By substituting the values of $q_{n}$ into Eq. (13), the equilibrium state is obtained.

\subsubsection{Equilibrium paths}

Since $q_{n}$ is equal to zero for even values of $n$, two types of solutions for $\alpha_{n}$ can be obtained. The first one is the symmetrical solution for which all coefficients $\alpha_{2 i}$ for $i=1,2, \ldots$ are zero, while in the asymmetrical solution, $\alpha_{2 j} \neq 0$ for some $j$, and all other $\alpha_{2 i}=0$ for $i=1,2, \ldots, i \neq j$. The reason of having only one non-zero asymmetrical mode in asymmetric equilibrium states will be discussed later.

On the symmetrical solution, from Eqs. (13) and (25), the mode coefficients $\alpha_{n}$ are as follows:

$$
\begin{cases}\alpha_{1}=-\frac{\lambda-h}{1+p} & \\ \alpha_{2 i+1}=\frac{(-1)^{i+1} \lambda}{(2 i+1)^{2}\left((2 i+1)^{2}+p\right)} & , i=1,2, \ldots \\ \alpha_{2 i}=0 & , i=1,2, \ldots\end{cases}
$$


By substituting the mode coefficients into Eq. (12), the value of $p$ is calculated:

$$
p=-\alpha \Delta T_{0}-\frac{h^{2}}{4}+\frac{(\lambda-h)^{2}}{4(1+p)^{2}}+\frac{\lambda^{2}}{4} \kappa_{2,2}(p),
$$

where, the function $\kappa_{M, N}(p)$ is defined in Appendix A. From this equation, the magnitude of $\lambda$ can be calculated as

$\lambda=\frac{h \pm \sqrt{(1+p)^{2}\left(4\left(p+\alpha \Delta T_{0}\right)\left(1+(1+p)^{2} \kappa_{2,2}(p)\right)+h^{2}\left(1+p(2+p) \kappa_{2,2}(p)\right)\right)}}{1+(1+p)^{2} \kappa_{2,2}(p)}$.

By substituting (26) into Eq. (8), one can determine the displacement field of the shallow arch:

$$
\Delta u(\xi)=u(\xi)-u_{0}(\xi)=-\left(\frac{\lambda-h}{1+p}+h\right) \sin \xi+\lambda \sum_{i=1}^{\infty} \frac{(-1)^{i+1} \sin ((2 i+1) \xi)}{(2 i+1)^{2}\left((2 i+1)^{2}+p\right)} .
$$

Eqs. (28) and (29) give the equilibrium path in the space of $(u, \lambda)$ for different values of $p$. In particular, the displacement of the midpoint $(\xi=\pi / 2)$ for the symmetrical solution is

$$
\Delta u_{\mathrm{Mid}}=\Delta u(\pi / 2)=-\left(\frac{\lambda-h}{1+p}+h\right)-\lambda \kappa_{2,1}(p) .
$$

Figure 3 illustrates the equilibrium path for four different values of $h$ and $\alpha \Delta T_{0}$. In this figure, the black curves are the primary equilibrium paths given by Eqs. (28) and (30). To verify the results, a finite element analysis (using a cylindrical arc-length method $[27,28]$ ) was also utilized to trace the equilibrium path from the initial unloaded state (points marked with $(\times)$ in Figure 3). For this purpose, 60 modified 2D Timoshenko beam elements with large transversal displacements are applied to discretize the arch. As it can be seen, the proposed method and the FEM give the same results.

On asymmetrical equilibrium paths, there exists a non-zero asymmetric mode coefficient $\left(\alpha_{2 j}\right)$. As a result, by considering the $2 j^{\text {th }}$ equation of (13), $p=-(2 j)^{2}$ along the corresponding asymmetrical solution. After substituting this value into the $2 i^{\text {th }}$ equation of (13) for $i=1,2, \ldots, i \neq j$, it can be concluded that $\alpha_{2 i}=0$. Subsequently, if $p$ is replaced with $-(2 j)^{2}$ in Eq. (26), 

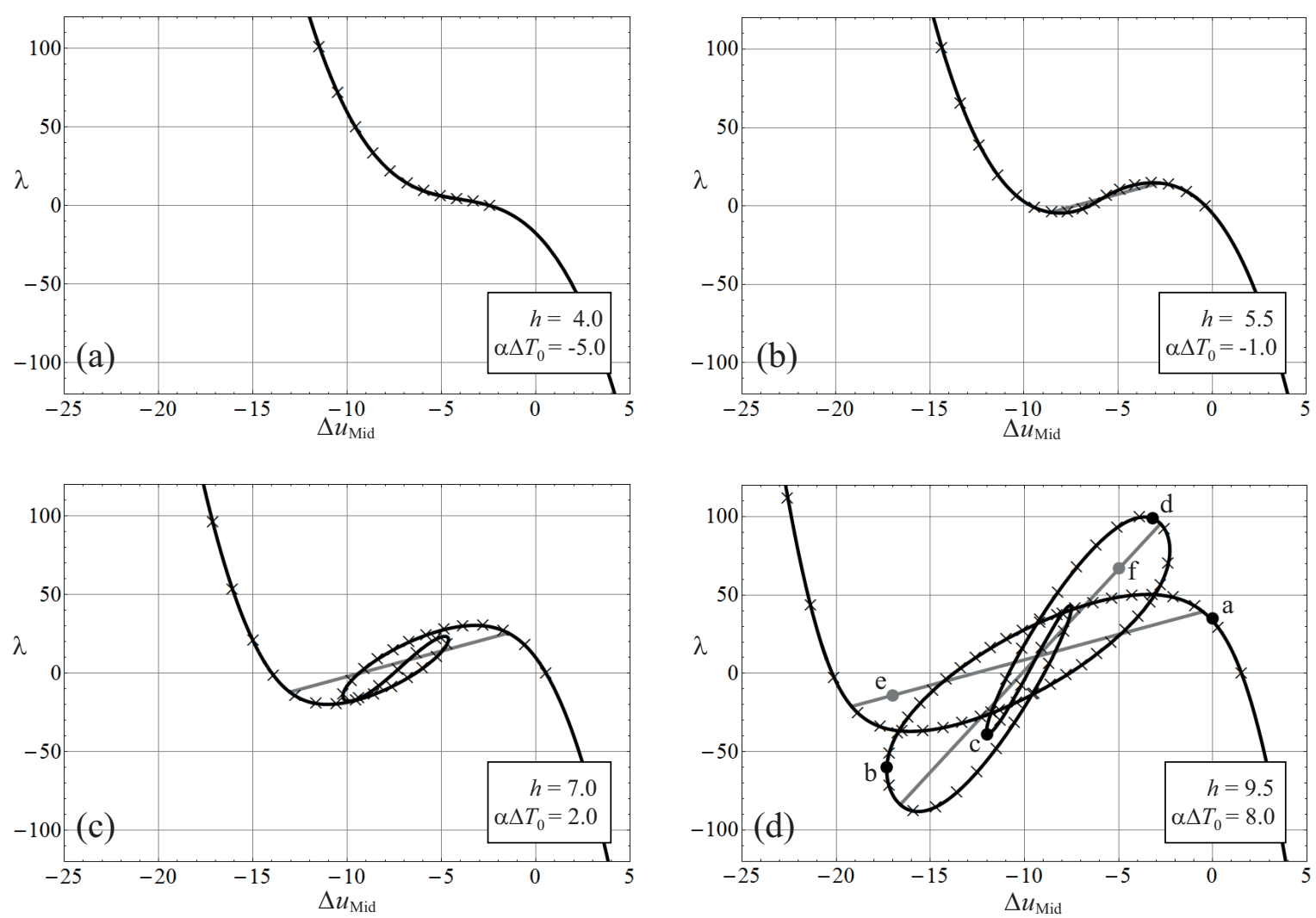

Figure 3: Equilibrium paths for four different values of $h$ and $\alpha \Delta T_{0}$

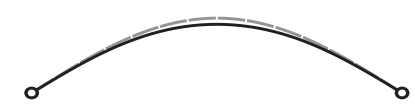

(a)

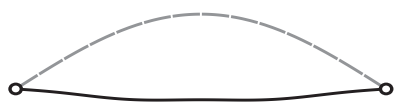

(c)

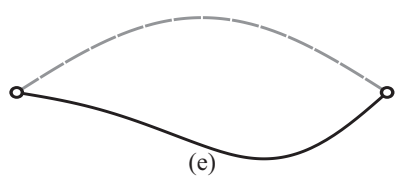

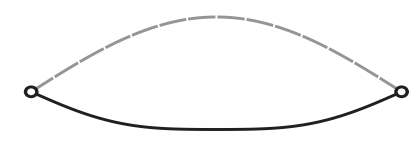

(b)

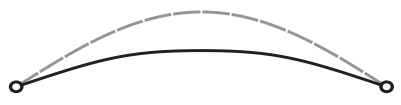

(d)

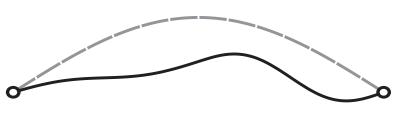

(f)

Figure 4: Equilibrium states corresponding to the points a-f shown in Figure 3(d) 
all even mode coefficients (except for $\alpha_{2 j}$ ) on the $2 j^{\text {th }}$ asymmetrical equilibrium path are $0 . \alpha_{2 j}$ can be explicitly computed from Eq. (27):

$\alpha_{2 j}= \pm \frac{1}{j} \sqrt{\alpha \Delta T_{0}+\frac{h^{2}}{4}-(2 j)^{2}-\frac{(\lambda-h)^{2}}{4\left(1-(2 j)^{2}\right)^{2}}-\frac{\lambda^{2}}{4} \kappa_{2,2}\left(-(2 j)^{2}\right)}, j=1,2, \ldots$

Similar to the symmetrical solution, the displacements of the shallow arch relative to the $j^{\text {th }}$ asymmetric equilibrium path are obtained by substituting the mode coefficients into Eq. (8):

$$
\begin{aligned}
\Delta u_{j}(\xi)= & -\left(\frac{\lambda-h}{1-(2 j)^{2}}+h\right) \sin \xi+\alpha_{2 j} \sin 2 j \xi \\
& +\lambda \sum_{i=1}^{\infty} \frac{(-1)^{i+1} \sin ((2 i+1) \xi)}{(2 i+1)^{2}\left((2 i+1)^{2}-(2 j)^{2}\right)}, j=1,2, \ldots
\end{aligned}
$$

This equation shows a linear relationship between $\Delta u$ and $\lambda$. Note that all values of $\lambda$ that result in real values for $\alpha_{2 j}$ (Eq. (31)) are acceptable for asymmetric equilibrium paths in the space of $(u, \lambda)$. By replacing $\xi$ with $\pi / 2$, one can obtain the displacement of the midpoint in the $j^{\text {th }}$ asymmetrical solution:

$$
\Delta u_{j \mathrm{Mid}}=-\left(\frac{\lambda-h}{1-(2 j)^{2}}+h\right)-\lambda \kappa_{2,1}\left(-(2 j)^{2}\right), j=1,2, \ldots
$$

The gray lines in Figure 3 display the asymmetric paths calculated by Eq. (33).

The equilibrium states corresponding to the points a-f shown in Figure 3(d) are shown in Figure 4. The equilibrium states a-d are symmetric, while the states $\mathbf{e}$ and $\mathbf{f}$ belong to the first and the second asymmetric equilibrium paths, respectively.

In Section 4, it is proven that the natural frequency corresponding to $\alpha_{n}=0$ is equal to $n \sqrt{n^{2}+p}$. Consequently, on the symmetrical solution, the natural frequency relative to the $i^{\text {th }}$ asymmetrical mode coefficients $\left(\alpha_{2 i}\right)$ is a function of $p$ and equals $2 i \sqrt{(2 i)^{2}+p}$. On the $j^{\text {th }}$ asymmetric equilibrium path, since $p=-(2 j)^{2}$ along the path, $\omega_{2 i}$ is constant and equal to $2 i \sqrt{(2 i)^{2}-(2 j)^{2}}$ for $i=1,2, \ldots, i \neq j$. In order to find the natural frequencies corresponding to non-zero mode coefficients, Eq. (23) is solved numerically. Note that a complex root for $\omega_{n}$ shows the instability of the shallow arch in the direction of $\sin n \xi$. 


\subsubsection{Critical points}

This section focuses attention on determining the critical points of half-sine shallow arches under a concentrated loading at the midpoint, i.e., the equilibrium points at which the constraint (17) is satisfied. It can be easily proven that all asymmetric equilibrium paths include critical points only at the intersection of symmetrical and asymmetrical solutions, i.e., at the bifurcation points. In order to prove this, the properties of equilibrium points on asymmetrical solution are examined. Since $p=-(2 j)^{2}$ on the $j^{\text {th }}$ asymmetric equilibrium path, $\beta_{k k}=0$ for $k=2 j$ (Eq. (16)), while $\beta_{k k} \neq 0$ for $k=1,2, \ldots, k \neq 2 j$. From Eq. (17), $\alpha_{2 j}=0$, which means that only symmetrical mode coefficients can be non-zero at critical points. In other words, all critical points (limit and bifurcation points) are located on the symmetric equilibrium paths. Similarly, for an arbitrary loading, it can be proven that all critical points are always located on the primary equilibrium path(s), and bifurcated paths include critical points only at their intersection with the primary equilibrium path(s).

The critical condition (17) can be satisfied in two ways. The first case corresponds to $p=-n^{2}$ for some $n$. Under this condition, $\beta_{n n}$ becomes zero, and subsequently, $\alpha_{n}$ should have a null value to satisfy (17). This case will be discussed later. If $p \neq-n^{2}$ for $n=1,2, \ldots$, Eq. (17) can be rewritten in a simpler form:

$$
1+\sum_{n=1}^{\infty} \frac{\alpha_{n}^{2}}{\beta_{n n}}=0
$$

By substituting the values of $\alpha_{n}$ given from Eq. (26) into (34), the following equation is obtained:

$$
1+\frac{\left(\lambda_{\mathrm{Cr}}-h\right)^{2}}{2(1+p)^{3}}+\frac{\lambda_{\mathrm{Cr}}{ }^{2}}{2} \kappa_{2,3}(p)=0
$$

where, $\lambda_{\mathrm{Cr}}$ is the dimensionless critical load parameter. Eqs. (27) and (35) describe the locus of limit points as a set of surfaces in the space of $\left(h, \alpha \Delta T_{0}, \lambda_{\mathrm{Cr}}\right)$. Figure 5 shows these surfaces over $\left\{\left(h, \alpha \Delta T_{0}\right) \in \mathbb{R}^{2} \mid 0<h<10,-10<\alpha \Delta T_{0}<\right.$ $10\}$. The displacements corresponding to the critical state can be obtained using Eq. (29). For a specific pair of $\left(h, \alpha \Delta T_{0}\right)$, there can be zero, two, four or more 
values for $\lambda_{\mathrm{Cr}}$, corresponding to limit points on the relative equilibrium path (Figure 3). The surface 1 corresponds to the first and the last limit points on the equilibrium path, while the other surfaces denote the locus of limit points between them.

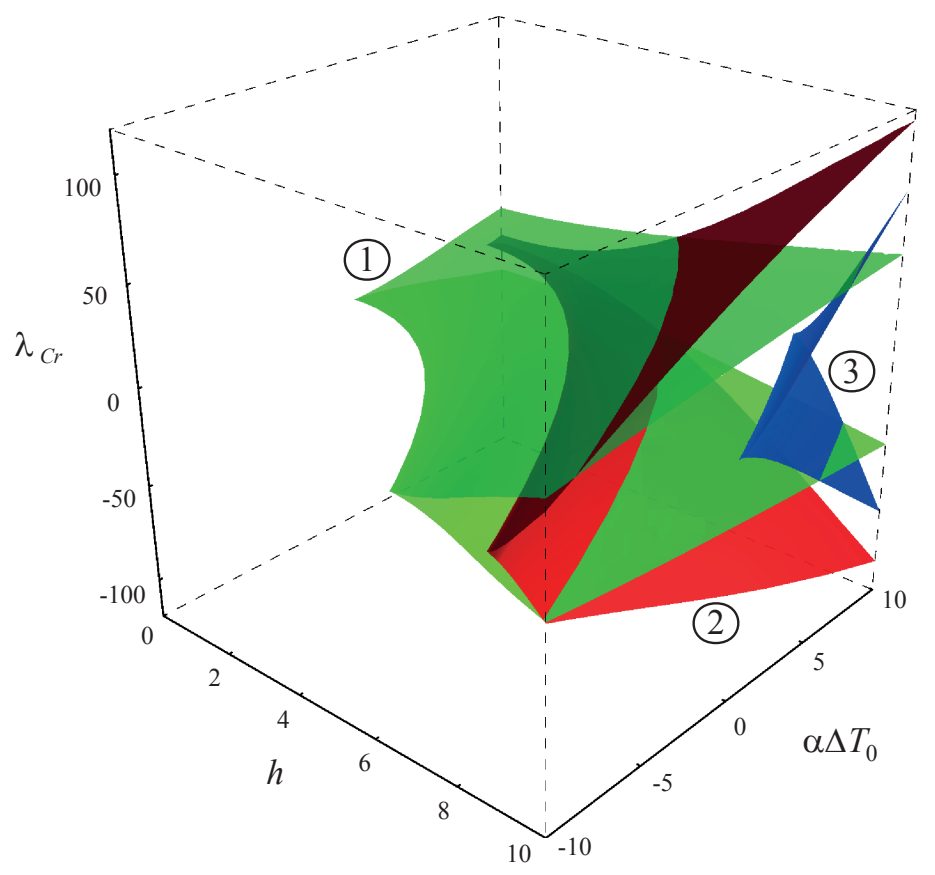

Figure 5: The locus of limit points in the space of $\left(h, \alpha \Delta T_{0}, \lambda_{\mathrm{Cr}}\right)$

By projecting these surfaces on the plane of $h$ and $\alpha \Delta T_{0}$, one can isolate areas within this 2-parameter space that correspond to geometries and initial temperature values leading to different number of limit points on the equilibrium path (Figure 6). The boundaries separating areas with different number of limit points are denoted by $B_{j}$ for $j=1,2, \ldots$ It can be proven that at the $j^{\text {th }}$ boundary $B_{j}$, the value of $p$ is equal to $-(2 j-1)^{2}$. Subsequently, $\beta_{2 j-1}=\alpha_{2 j-1}=0$ from Eqs. (16) and (17). By substituting the value of $p$ in the $j^{\text {th }}$ equation of (13), it is shown that $q_{j}=0$. In the case of $j=1, \lambda_{\mathrm{Cr}}$ is equal to $h$. From Eq. (27) and using $\kappa_{2,2}(-1)=\left(7 \pi^{2}-69\right) / 48$ the first 
boundary is determined as:

$$
B_{1}=\left\{\left(h, \alpha \Delta T_{0}\right) \in \mathbb{R}^{2} \mid \alpha \Delta T_{0}=1+\frac{7 \pi^{2}-117}{192} h^{2}\right\}
$$

For $j \geq 2, \lambda_{\mathrm{Cr}}$ is equal to zero. As a result, $\alpha_{n}=0$ for $n=2,3, \ldots$ from Eq. (13). By substituting the values of $\alpha_{n}$ into Eq. (27), the $j^{\text {th }}$ boundary $B_{j}$ is obtained:

$B_{j}=\left\{\left(h, \alpha \Delta T_{0}\right) \in \mathbb{R}^{2} \mid \alpha \Delta T_{0}=(2 j-1)^{2}+\left(\frac{1}{64 j^{2}(j-1)^{2}}-\frac{1}{4}\right) h^{2}\right\}, j=2,3, \ldots$

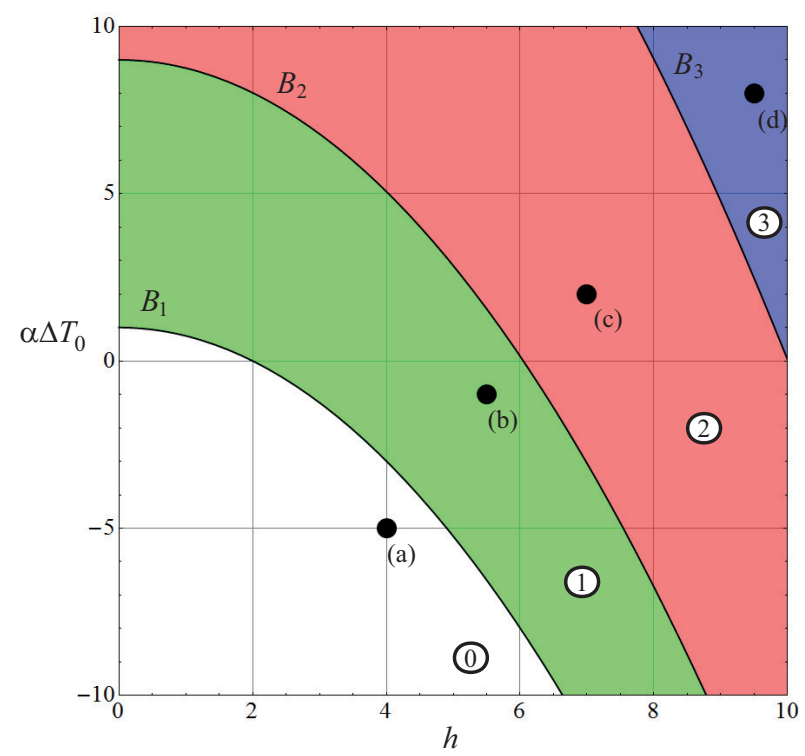

Figure 6: Areas with different number of limit points: zero (area 0), two (area 1), four (area 2) and six (area 3) limit points

Figure 6 shows the $B_{1}, B_{2}$ and $B_{3}$ boundaries for the interval $\left\{\left(h, \alpha \Delta T_{0}\right) \in\right.$ $\left.\mathbb{R}^{2} \mid 0<h<10,-10<\alpha \Delta T_{0}<10\right\}$. Four areas are displayed in this figure. For the shallow arches with $h$ and $\alpha \Delta T_{0}$ in the white area, for example point (a), there is no limit point on their corresponding equilibrium paths. For the values of $h$ and $\alpha \Delta T_{0}$ located in the areas 1,2 and 3, there are two, four and six limit points, respectively. The equilibrium paths corresponding to the points (a)-(d) are shown in Figure 3. For the values of $h$ and $\alpha \Delta T_{0}$ on the boundaries $B_{j}$, the two limit points and the inflection point coincide. 
Recall that bifurcation points are found at the intersection of branches of symmetrical and asymmetrical solutions. Consequently, the bifurcation point relative to the $j^{\text {th }}$ asymmetric equilibrium path should satisfy the following properties:

$$
\left\{\begin{array}{l}
\alpha_{2 i}=0 \quad, i=1,2, \ldots \\
p=-(2 j)^{2}
\end{array}\right.
$$

In contrast with other equilibrium points on asymmetrical solutions, the $j^{\text {th }}$ bifurcation point satisfies the additional constraint $\alpha_{2 j}=0$. As a result, a direct relationship between $h, \alpha \Delta T_{0}$ and $\lambda_{\mathrm{Cr}}$ is obtained by considering Eq. (31):

$C_{j}=\alpha \Delta T_{0}+\frac{h^{2}}{4}-(2 j)^{2}-\frac{\left(\lambda_{\mathrm{Cr}}-h\right)^{2}}{4\left(1-(2 j)^{2}\right)^{2}}-\frac{\lambda_{\mathrm{Cr}}^{2}}{4} \kappa_{2,2}\left(-(2 j)^{2}\right)=0, j=1,2, \ldots$

Eq. (39) describes a number of surfaces $C_{j}\left(h, \alpha \Delta T_{0}, \lambda_{\mathrm{Cr}}\right)=0$ in the space of $\left(h, \alpha \Delta T_{0}, \lambda_{\mathrm{Cr}}\right)$. These surfaces display the value of critical loads $\lambda_{\mathrm{Cr}}$ corresponding to the bifurcation points as a function of the parameters $h$ and $\alpha \Delta T_{0}$. Figure 7 shows the mentioned surfaces for the interval $\left\{\left(h, \alpha \Delta T_{0}\right) \in \mathbb{R}^{2} \mid 0<\right.$ $\left.h<10,-10<\alpha \Delta T_{0}<10\right\}$.

In what follows, the surface $C_{i}\left(h, \alpha \Delta T_{0}, \lambda_{\mathrm{Cr}}\right)=0$ will be referred to as surface $i$ and identified on the figures with the label (i). For a specific pair of $\left(h, \alpha \Delta T_{0}\right)$, the surface 1 displays the magnitude of the critical loads at the first and the last bifurcation points of the equilibrium path. The projection of the surfaces on the plane of $h$ and $\alpha \Delta T_{0}$ identifies a number of boundaries represented by $B_{j}^{\prime}$. These boundaries separate areas with different number of bifurcation points along the equilibrium path. Since $C_{j}$ is continuous and differentiable, the following constraint should be satisfied:

$$
\frac{\partial C_{j}}{\partial \lambda_{\mathrm{Cr}}}=\frac{1}{2}\left(\lambda_{\mathrm{Cr}} \kappa_{2,2}\left(-(2 j)^{2}\right)+\frac{\lambda_{\mathrm{Cr}}-h}{\left(1-(2 j)^{2}\right)^{2}}\right)=0, j=1,2, \ldots
$$

By calculating $\lambda_{\mathrm{Cr}}$ from (40) and substituting its value into Eq. (39), the 


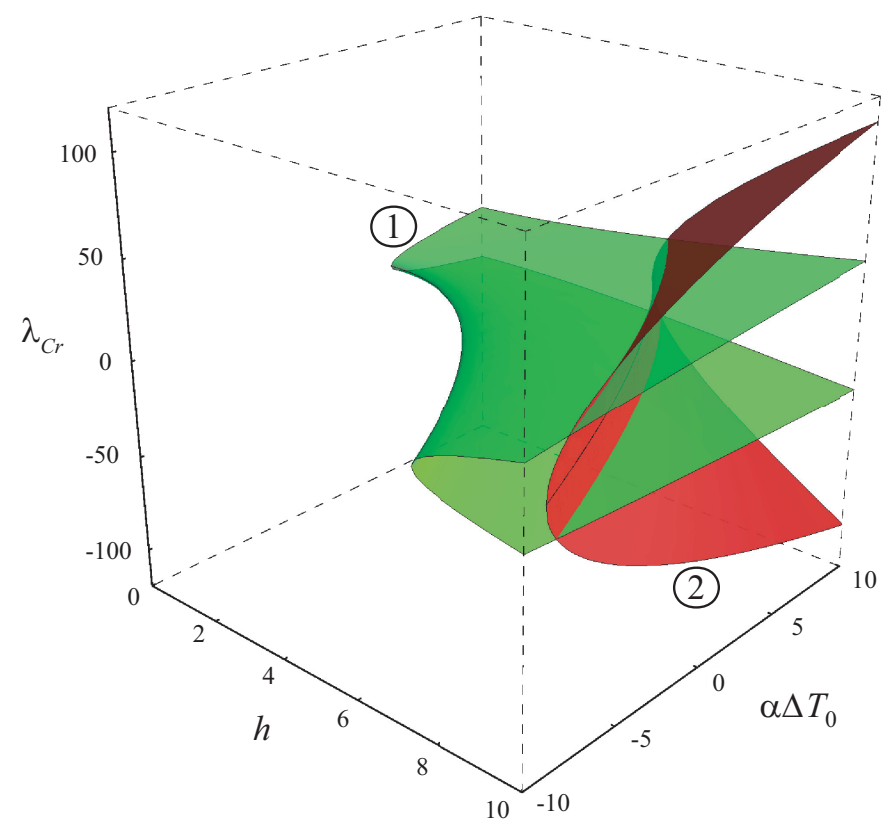

Figure 7: The locus of bifurcation points in the space of $\left(h, \alpha \Delta T_{0}, \lambda_{\mathrm{Cr}}\right)$

boundaries $B_{j}^{\prime}$ are obtained:

$B_{j}^{\prime}=\left\{\left(h, \alpha \Delta T_{0}\right) \in \mathbb{R}^{2} \mid \alpha \Delta T_{0}=4 j^{2}+\frac{2 j^{2}\left(1+32 j^{4}-32 j^{6}+j^{2}\left(-10-\frac{32}{3 \pi^{2}}\right)\right)}{\left(1-4 j^{2}\right)^{4}} h^{2}\right\}, j=1,2, \ldots$

Figure 8 displays different areas corresponding to different number of bifurcation points on equilibrium paths for the interval $\left\{\left(h, \alpha \Delta T_{0}\right) \in \mathbb{R}^{2} \mid 0<h<10,-10<\right.$ $\left.\alpha \Delta T_{0}<10\right\}$. The corresponding asymmetrical solutions for points (a)-(d) are shown in Figure 3 (gray lines).

A particular case, which can be of interest, is the coincidence of limit and bifurcation points on the equilibrium path. This corresponds to cases when the limit point constraint (35) and the bifurcation constraint (38) are satisfied simultaneously. By substituting the value of $p$ from (38) into (35) and considering Eq. (39), the following equations are obtained:

$$
\left\{\begin{array}{l}
1+\frac{\left(\lambda_{\mathrm{Cr}}-h\right)^{2}}{2\left(1-(2 j)^{2}\right)^{3}}+\frac{\lambda_{\mathrm{Cr}}^{2}}{2} \kappa_{2,3}\left(-(2 j)^{2}\right)=0 \quad, j=1,2, \ldots \\
C_{j}=0
\end{array}\right.
$$




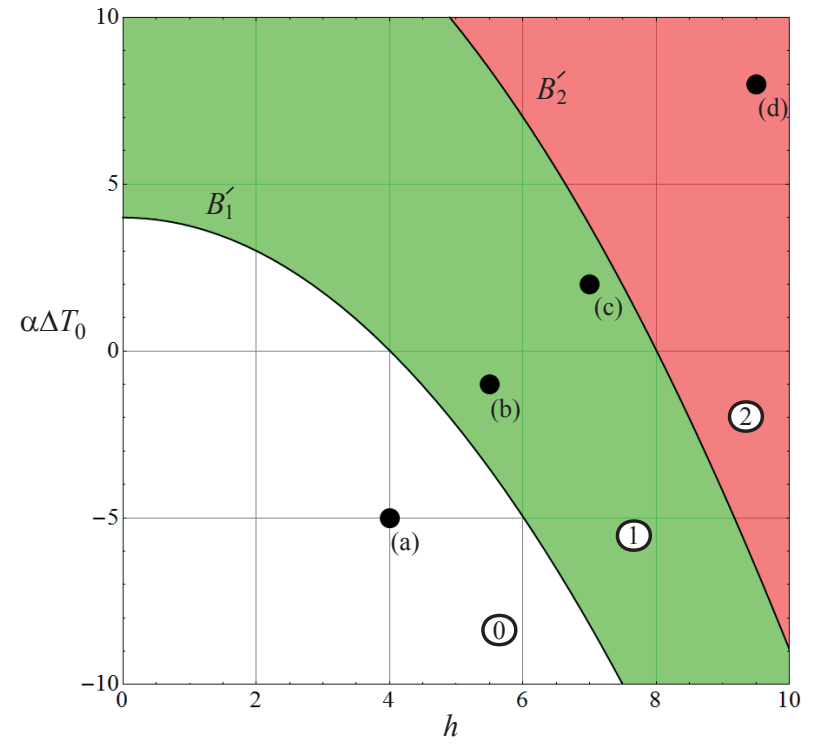

Figure 8: Areas with different number of bifurcation points: zero (area 0), two (area 1) and four (area 2) bifurcation points

For each $j$, Eq. (42) describes a relationship between $h, \alpha \Delta T_{0}$ and $\lambda_{\mathrm{Cr}}$. An exact solution can be obtained, for instance, Eqs. (43) and (44) denote the solutions for $j=1$ and $j=2$, respectively:

$$
\begin{gathered}
\left\{\begin{array}{l}
\lambda_{\mathrm{Cr}}=1.0247 h \pm \sqrt{55.3349+0.02533 h^{2}} \\
\alpha \Delta T_{0}=5.6-0.2481 h^{2} \pm h \sqrt{0.000759+3.4748 \times 10^{-7} h^{2}}
\end{array}\right. \\
\left\{\begin{array}{l}
\lambda_{\mathrm{Cr}}=0.5247 h \pm \sqrt{3541.43-0.2494 h^{2}} \\
\alpha \Delta T_{0}=22.4-0.2497 h^{2} \pm h \sqrt{0.000376-2.6492 \times 10^{-8} h^{2}}
\end{array}\right.
\end{gathered}
$$

Note that there are two solutions for each $j$. One corresponds to the coincidence of the first bifurcation point (the beginning of the $j^{\text {th }}$ asymmetrical solution) with a limit point. Here, this state is named $C_{j}^{T}$. The other solution corresponds to the coincidence of the second bifurcation point (the end of the $j^{\text {th }}$ asymmetrical solution path) with a limit point $\left(C_{j}^{B}\right)$. Figure 9 illustrates $C_{1}^{T}$, $C_{1}^{B}, C_{2}^{T}$ and $C_{2}^{B}$ calculated from Eqs. (43) and (44) in the space of $\left(h, \alpha \Delta T_{0}\right)$. In this figure, the solid curves are $C_{1}^{T}$ and $C_{2}^{T}$, the dashed curves are relative to $C_{1}^{B}$ and $C_{2}^{B}$. 


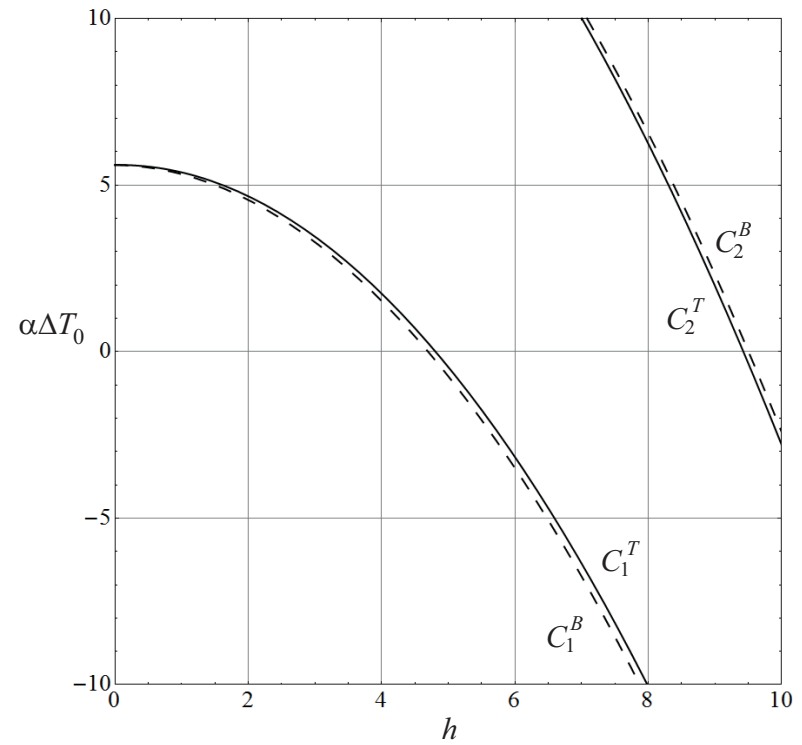

Figure 9: Locus of coincidence of limit and bifurcation points

An issue that could be of interest is the sequence of critical points along the equilibrium path. The parameters $h$ and $\alpha \Delta T_{0}$ can swap the sequence of the first limit point and the first bifurcation point on the equilibrium path. For a pair $h$ and $\alpha \Delta T_{0}$ on the curve $C_{1}^{T}$, the first limit and bifurcation points coincide. For the states below this curve, a limit point is first detected when tracing the equilibrium path. Conversely, for states above the curve $C_{1}^{T}$, a bifurcation point is the first critical point on the equilibrium path.

\subsection{Uniform loading}

In this example, the shallow arch under a uniform distributed loading is investigated (Figure $1(\mathrm{c})$ ). The value of non-dimensionalized load $Q_{2}$ is constant, with value $\lambda$ over the span. If, for convenience, the arbitrary scaling factor $c$ is assumed $\pi / 4$, then the value of $q_{n}$ for $n=1,2, \ldots$ becomes

$$
\begin{cases}q_{1}=\lambda-h & \\ q_{2 i+1}=\frac{\lambda}{2 i+1} & , i=1,2, \ldots \\ q_{2 i}=0 & , i=1,2, \ldots\end{cases}
$$




\subsubsection{Equilibrium paths}

Similarly to the case of the concentrated load, the arch has symmetrical and asymmetrical solutions. By considering Eqs. (13) and (45), the magnitude of mode coefficients in a symmetrical solution is

$$
\begin{cases}\alpha_{1}=-\frac{\lambda-h}{1+p} & \\ \alpha_{2 i+1}=\frac{-\lambda}{(2 i+1)^{3}\left((2 i+1)^{2}+p\right)} & , i=1,2, \ldots \\ \alpha_{2 i}=0 & , i=1,2, \ldots\end{cases}
$$

By substituting the computed $\alpha_{n}$ into Eq. (12), $p$ is obtained:

$$
p=-\alpha \Delta T_{0}-\frac{h^{2}}{4}+\frac{(\lambda-h)^{2}}{4(1+p)^{2}}+\frac{\lambda^{2}}{4} \kappa_{4,2}(p) .
$$

This equation is similar to $(27)$, except that $\kappa_{2,2}(p)$ is replaced by $\kappa_{4,2}(p)$. From Eq. (47), the load parameter $\lambda$ can be calculated as a function of $p, h$ and $\alpha \Delta T_{0}$. In addition, the dimensionless displacement field for the symmetric equilibrium path is obtained by substituting the values of $\alpha_{n}$ into Eq. (8):

$$
\Delta u(\xi)=-\left(\frac{\lambda-h}{1+p}+h\right) \sin \xi+\lambda \sum_{i=1}^{\infty} \frac{-\sin ((2 i+1) \xi)}{(2 i+1)^{3}\left((2 i+1)^{2}+p\right)} .
$$

The displacement of the midpoint is:

$$
\Delta u_{\mathrm{Mid}}=-\left(\frac{\lambda-h}{1+p}+h\right)-\lambda \kappa_{3,1}^{\prime}(p),
$$

where, $\kappa_{M, N}^{\prime}(p)$ is defined in Appendix A. Figure 10 shows the equilibrium paths of the shallow arch for four different values of $h$ and $\alpha \Delta T_{0}$. Black solid lines and the points marked with $(\times)$ denote the results computed by the proposed method and FEM, respectively.

In order to obtain the asymmetrical solution, the same procedure described in Section 5.1 can be followed. Eqs. (50) and (51) give the displacement field and the displacement of the midpoint for the $j^{\text {th }}$ asymmetric equilibrium path, respectively:

$$
\begin{aligned}
\Delta u_{j}(\xi)= & -\left(\frac{\lambda-h}{1-(2 j)^{2}}+h\right) \sin \xi+\alpha_{2 j} \sin 2 j \xi \\
& +\lambda \sum_{i=1}^{\infty} \frac{-\sin ((2 i+1) \xi)}{(2 i+1)^{3}\left((2 i+1)^{2}-(2 j)^{2}\right)}, j=1,2, \ldots
\end{aligned}
$$



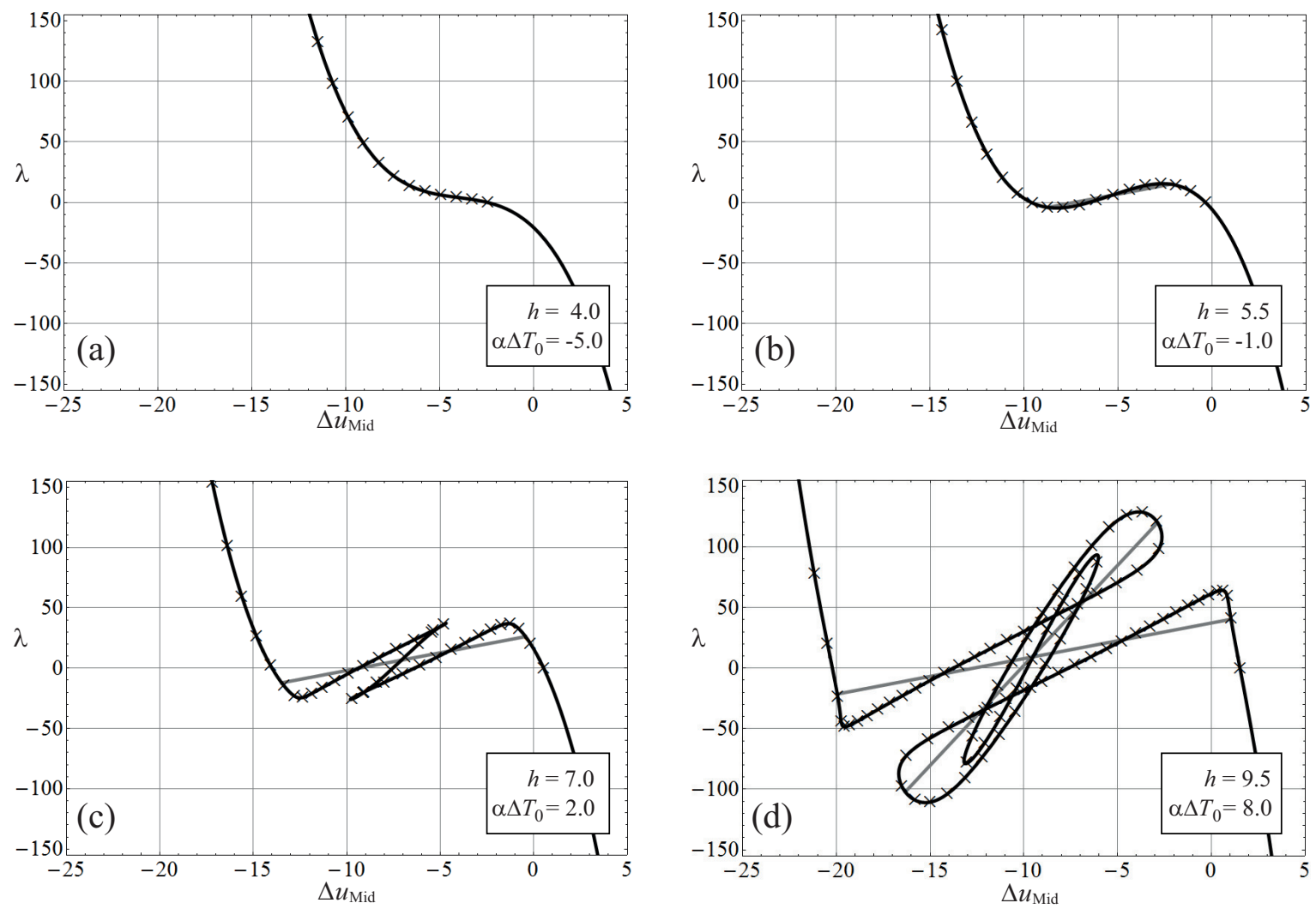

Figure 10: Equilibrium paths for four different values of $h$ and $\alpha \Delta T_{0}$

$$
\Delta u_{j \mathrm{Mid}}=-\left(\frac{\lambda-h}{1-(2 j)^{2}}+h\right)+\lambda \kappa_{3,1}^{\prime}\left(-(2 j)^{2}\right), j=1,2, \ldots
$$

Here, $\alpha_{2 j}$ is similar to Eq. (31), where $\kappa_{2,2}(p)$ is replaced by $\kappa_{4,2}(p)$. In Figure 10, the asymmetric equilibrium paths are shown by gray solid lines. To estimate natural frequencies in the vicinity of an equilibrium state, the same procedure utilized in the concentrated loading problem (Section 5.1) can be followed.

\subsubsection{Critical points}

From Eq. (45), it is derived that $q_{2 k}=0$ for the uniformly distributed loading. Recall that critical points are always located on the equilibrium path on which the value of all $\alpha_{n}$ corresponding to $q_{n}=0$ for some $n$ equals zero. Consequently, the locus of limit and bifurcation points is included into the 
symmetrical solution. If $p \neq-n^{2}$ for $n=1,2, \ldots$, the critical condition (34) can be written in the following form:

$$
1+\frac{\left(\lambda_{\mathrm{Cr}}-h\right)^{2}}{2(1+p)^{3}}+\frac{\lambda_{\mathrm{Cr}}^{2}}{2} \kappa_{4,3}(p)=0
$$

Eqs. (47) and (52) provide the locus of limit points as a set of surfaces in the space of $\left(h, \alpha \Delta T_{0}, \lambda_{\mathrm{Cr}}\right)$. Figure 11 shows these surfaces for the interval $\left\{\left(h, \alpha \Delta T_{0}\right) \in \mathbb{R}^{2} \mid 0<h<10,-10<\alpha \Delta T_{0}<10\right\}$. This figure shows that, by increasing $h$ and $\alpha \Delta T_{0}$, the number of pairs of limit points on the symmetric equilibrium path increases. The boundaries of domains with different number of limit points correspond to $p=-(2 j-1)^{2}$ for $j=1,2, \ldots$. By considering the $j^{\text {th }}$ equation of (13), it is concluded that $q_{j}=0$. Consequently, $\lambda_{\mathrm{Cr}}=h$ and $\lambda_{\mathrm{Cr}}=0$ for $j=1$ and $j \geq 2$, respectively. By substituting the values of $p$ and $\lambda_{\mathrm{Cr}}$ into Eq. (47), the corresponding boundaries $B_{j}$ are determined. Note that the computed boundaries for $j \geq 2$ coincide with the boundaries defined by Eq. (37) for the concentrated load case. For $j=1, B_{1}$ is

$$
B_{1}=\left\{\left(h, \alpha \Delta T_{0}\right) \in \mathbb{R}^{2} \mid \alpha \Delta T_{0}=1+\frac{\pi^{4}+26 \pi^{2}-450}{384} h^{2}\right\} .
$$

The constraints (38) should be satisfied at the bifurcation points on the $j^{\text {th }}$ asymmetric equilibrium path. By substituting the value of $p$ into (47), a direct relationship between $h, \alpha \Delta T_{0}$ and $\lambda_{\mathrm{Cr}}$ is obtained:

$C_{j}=\alpha \Delta T_{0}+\frac{h^{2}}{4}-(2 j)^{2}-\frac{\left(\lambda_{\mathrm{Cr}}-h\right)^{2}}{4\left(1-(2 j)^{2}\right)^{2}}-\frac{\lambda_{\mathrm{Cr}}^{2}}{4} \kappa_{4,2}\left(-(2 j)^{2}\right)=0, j=1,2, \ldots$

Figure 12 shows $C_{1}$ and $C_{2}$ (the surfaces 1 and 2, respectively) for the interval $\left\{\left(h, \alpha \Delta T_{0}\right) \in \mathbb{R}^{2} \mid 0<h<10,-10<\alpha \Delta_{0} T<10\right\}$. In order to find the boundaries of domains with different number of bifurcation points along the symmetric equilibrium path, the constraint $\partial C_{j} / \partial \lambda_{\mathrm{Cr}}=0$ is added to Eq. (54); after eliminating $\lambda_{\mathrm{Cr}}$, the boundaries $B_{j}^{\prime}$ are determined.

The specific configurations that cause the coincidence of a limit point and a bifurcation point on the equilibrium path are obtained when the limit point constraint (52) and the bifurcation constraint (54) are satisfied simultaneously. 


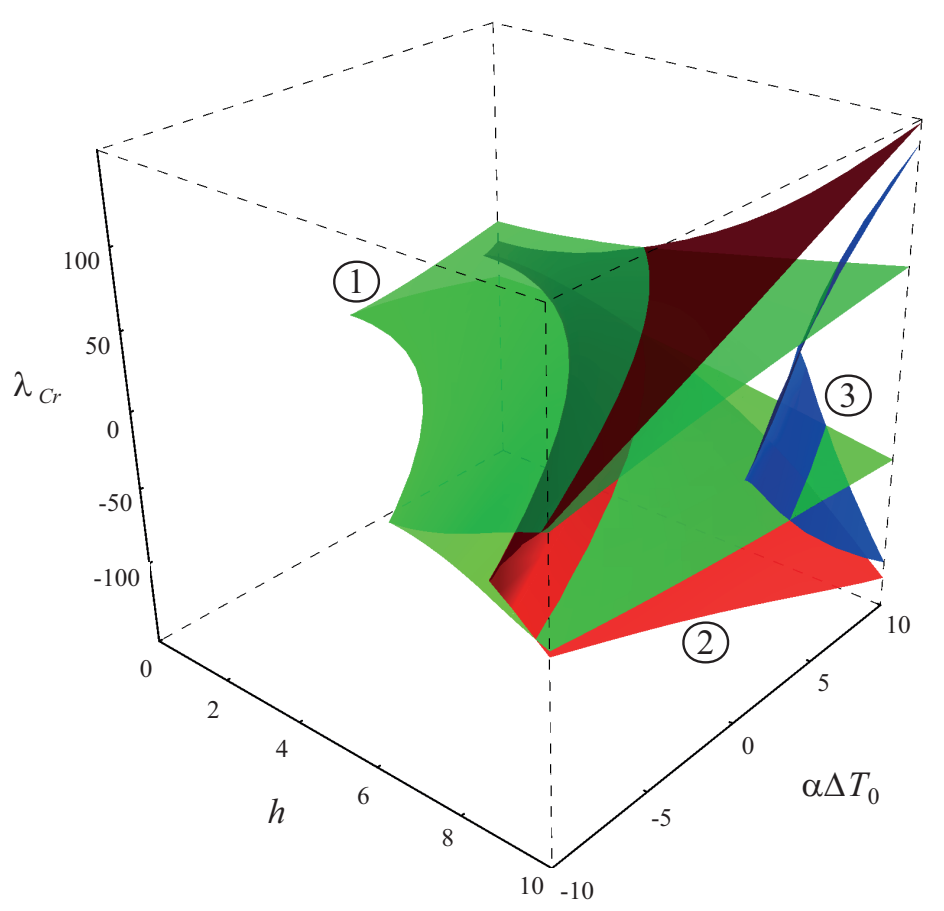

Figure 11: The locus of limit points in the space of $\left(h, \alpha \Delta T_{0}, \lambda_{\mathrm{Cr}}\right)$

The value of $p$ for the coincidence of a limit point and the bifurcation point corresponding to $j^{\text {th }}$ asymmetrical solution is equal to $-(2 j)^{2}$. As an example, Eq. (55) denotes the solution for $j=1$ :

$$
\left\{\begin{array}{l}
\lambda_{\mathrm{Cr}}=1.0027 h \pm \sqrt{54.1446+0.002686 h^{2}} \\
\alpha \Delta T_{0}=5.5108-0.2498 h^{2} \pm h \sqrt{8.5908 \times 10^{-6}+4.2614 \times 10^{-10} h^{2}}
\end{array}\right.
$$

This means that if $h$ and $\alpha \Delta T_{0}$ satisfy the second equation of (55), the first asymmetric equilibrium path intersects the symmetrical solution at a limit point with $\lambda_{\mathrm{Cr}}$ given in Eq. (55). Similar arguments can be made for $j \geq 2$. Eq. (56) provides the solution to $j=2$ :

$$
\left\{\begin{array}{l}
\lambda_{\mathrm{Cr}}=0.8976 h \pm \sqrt{6059.09-0.09188 h^{2}} \\
\alpha \Delta T_{0}=23.1445-0.2500 h^{2} \mp h \sqrt{0.0000672-1.0192 \times 10^{-9} h^{2}}
\end{array}\right.
$$

Similar to the case of the concentrated loading, the sequence of the first limit and bifurcation points on the equilibrium path is a function of the parameters 


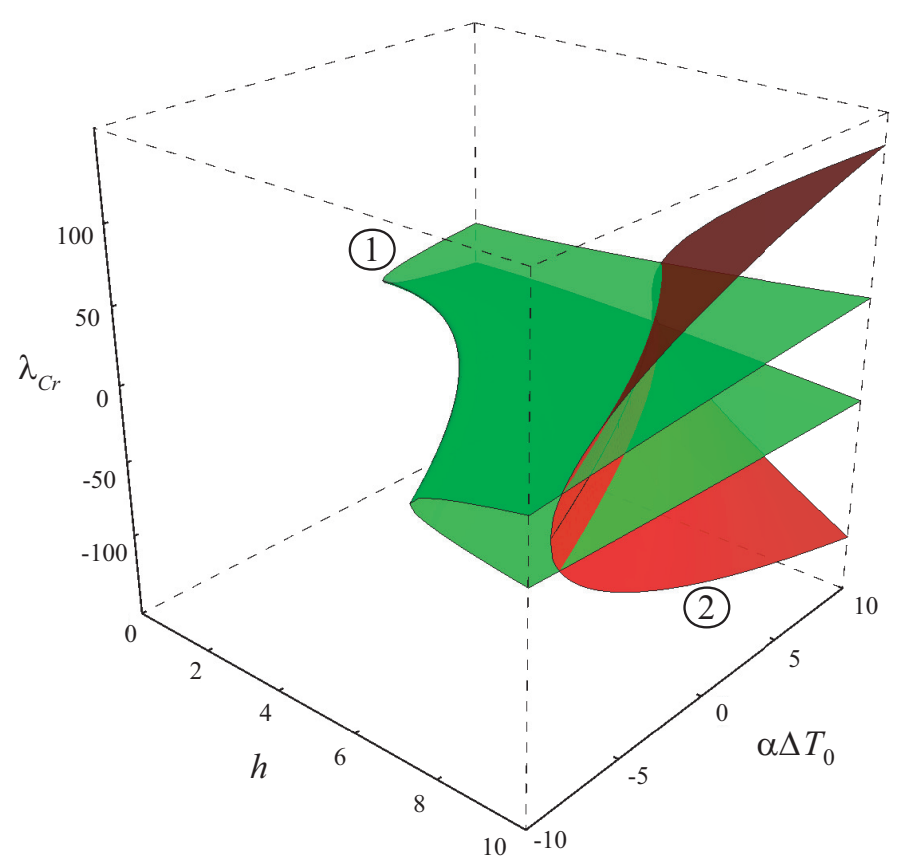

Figure 12: The locus of bifurcation points in the space of $\left(h, \alpha \Delta T_{0}, \lambda_{\mathrm{Cr}}\right)$

$h$ and $\alpha \Delta T_{0}$. For the values below the coincidence curve of the first critical points, a limit point is detected first, and for higher values, an inverse sequence is observed.

\subsection{Asymmetrical uniform loading}

In this section, the shallow arch under asymmetrical uniform loading is investigated (Figure $1(\mathrm{~d})$ ). The non-dimensionalized load $Q_{3}$ can be written as a function of the load parameter $\lambda$ :

$$
Q_{3}=\lambda(1-2 H(\pi / 2)),
$$


where $H$ is the Heaviside step function. If the scaling factor $c$ is assumed to be $\pi / 4$, then

$$
\begin{cases}q_{1}=-h & \\ q_{2 i+1}=0 & , i=1,2, \ldots \\ q_{4 i-2}=\frac{\lambda}{2 i-1} & , i=1,2, \ldots \\ q_{4 i}=0 & , i=1,2, \ldots\end{cases}
$$

\subsubsection{Equilibrium paths}

The mode coefficients $\alpha_{n}$ can be calculated by substituting the values of $q_{n}$ from Eq. (58) into Eq. (13). For this type of loading, three types of equilibrium paths are obtained. At the equilibrium state Type I, the value of all $\alpha_{n}$ corresponding to $q_{n}=0$ is equal to zero:

$$
\begin{cases}\alpha_{1}=\frac{h}{1+p} & \\ \alpha_{2 i+1}=0 & , i=1,2, \ldots \\ \alpha_{4 i-2}=\frac{-2 \lambda}{(4 i-2)^{3}\left((4 i-2)^{2}+p\right)} & , i=1,2, \ldots \\ \alpha_{4 i}=0 & , i=1,2, \ldots\end{cases}
$$

which means that the displacement of the arch consists of mode shapes $\sin \xi$ and $\sin (4 i-2) \xi$ for $i=1,2, \ldots$ From the mode coefficients, the value of $p$ is calculated from Eq. (12):

$$
p=-\alpha \Delta T_{0}-\frac{h^{2}}{4}+\frac{h^{2}}{4(1+p)^{2}}+\frac{\lambda^{2}}{4} \kappa_{4,2}^{\prime \prime}(p),
$$

where, the function $\kappa_{M, N}^{\prime \prime}(p)$ is defined in Appendix A. From this equation, the load coefficient $\lambda$ is obtained as a function of $p, h$ and $\alpha \Delta T_{0}$. In addition, the displacement field can be determined by substituting the values of $\alpha_{n}$ into Eq. (8):

$$
\Delta u(\xi)=\left(\frac{-p}{1+p} h\right) \sin \xi+\lambda \sum_{i=1}^{\infty} \frac{-2 \sin ((4 i-2) \xi)}{(4 i-2)^{3}\left((4 i-2)^{2}+p\right)}
$$

As a result, the displacement of the midpoint $(\xi=\pi / 2)$ is

$$
\Delta u_{\mathrm{Mid}}=\frac{-p}{1+p} h
$$


This relationship indicates that $\lim _{p \rightarrow+\infty} \Delta u_{\mathrm{Mid}}=-h$. Figure 13 shows the equilibrium paths for four different values of $h$ and $\alpha \Delta T_{0}$. In this figure, the black solid curves represent the equilibrium paths obtained by the proposed procedure (Eqs. (60) and (62) for different values of $p$ ). For verification, the equilibrium path is also traced by finite element method with $602 \mathrm{D}$ Timoshenko beam elements (considering large transversal displacements) from the initial unloaded state (the points marked with $(\times)$ ).
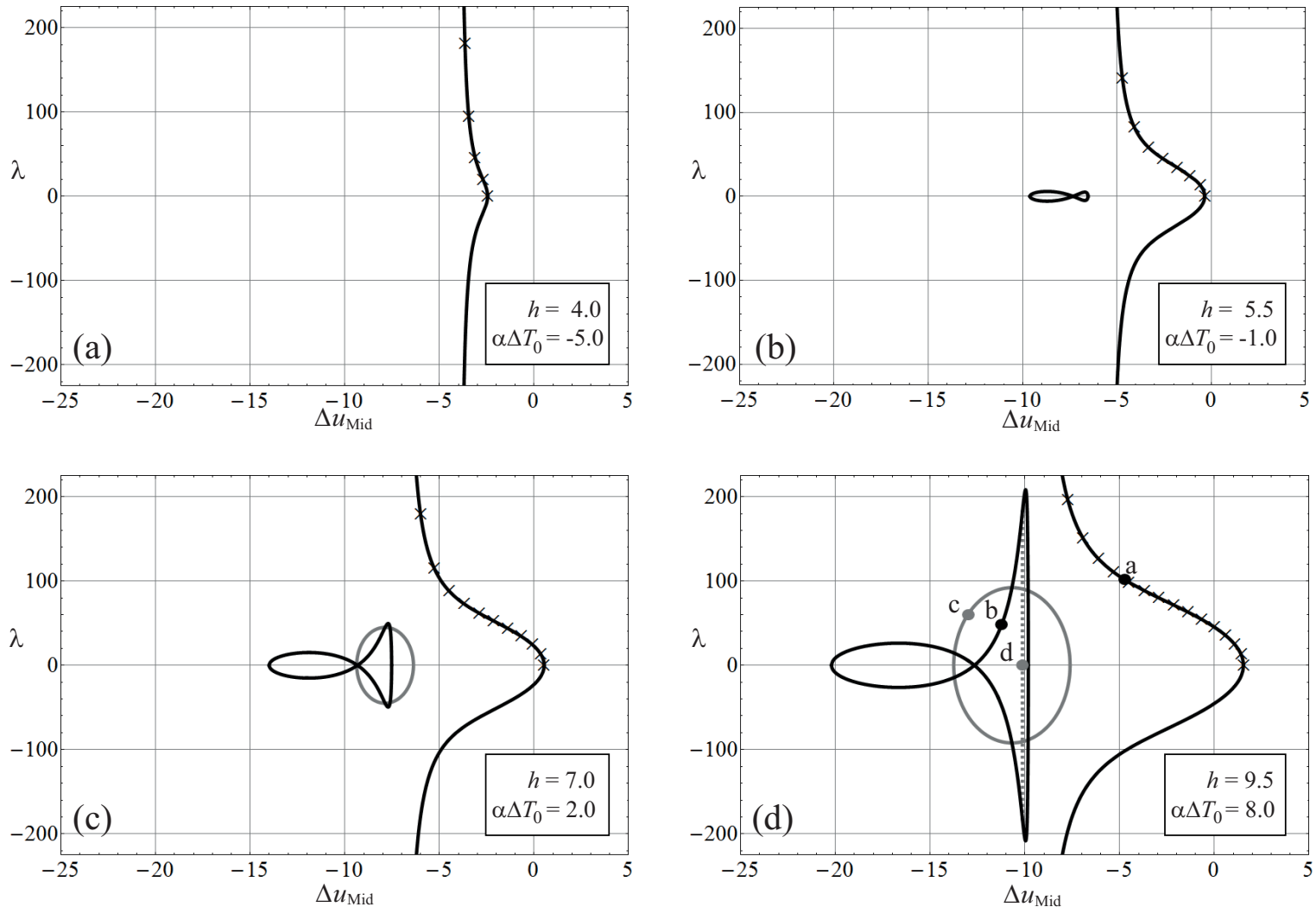

Figure 13: Equilibrium paths for four different values of $h$ and $\alpha \Delta T_{0}$

For sufficiently large values of $h$ and $\alpha \Delta T_{0}$ there is a secondary equilibrium path that does not pass through the initial unloaded equilibrium state (Figure 13(b-d)). Although the primary equilibrium path has no critical point, the secondary path includes limit (and bifurcation) points. Figure 14 illustrates the 
deformed shape of the shallow arch at the points a-d in Figure 13(d).

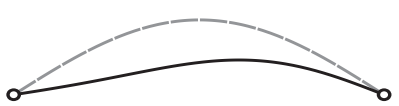

(a)

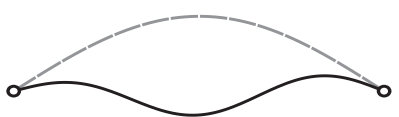

(c)

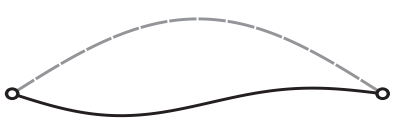

(b)

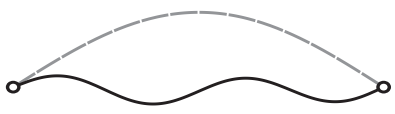

(d)

Figure 14: Equilibrium states corresponding to the points a-d shown in Figure 13(d)

From the relationships given in Section 4, the natural frequencies corresponding to the mode coefficients $\alpha_{2 i+1}$ and $\alpha_{4 i-2}$ for $i=1,2, \ldots$ are, respectively, equal to $(2 i+1) \sqrt{(2 i+1)^{2}+p}$ and $(4 i-2) \sqrt{(4 i-2)^{2}+p}$. To find the natural frequencies relative to non-zero mode coefficients, Eq. (23) is solved numerically.

At the equilibrium state Type II, $\alpha_{2 j+1} \neq 0$ for some values of $j$, i.e., the displacement field of the arch is a combination of all mode shapes existing in the Type I solution and the symmetric mode shape $\sin (2 j+1) \xi$ :

$$
\Delta u_{j^{\prime}}(\xi)=\left(\frac{j^{\prime 2}}{1-j^{\prime 2}} h\right) \sin \xi+\alpha_{j^{\prime}} \sin j^{\prime} \xi+\lambda \sum_{i=1}^{\infty} \frac{-2 \sin ((4 i-2) \xi)}{(4 i-2)^{3}\left((4 i-2)^{2}-j^{\prime 2}\right)},
$$

where, $j^{\prime}$ equals $2 j+1$ for $j=1,2, \ldots$. By computing $\alpha_{1}$ and $\alpha_{4 i-2}$ for $i=$ $1,2, \ldots$ from Eq. (13) and substituting their values the results into (12), $\alpha_{j^{\prime}}$ is calculated:

$$
\alpha_{j^{\prime}}= \pm \frac{2}{j^{\prime}} \sqrt{\alpha \Delta T_{0}+\frac{h^{2}}{4}-j^{\prime 2}-\frac{h^{2}}{4\left(1-j^{\prime 2}\right)^{2}}-\frac{\lambda^{2}}{4} \kappa_{4,2}^{\prime \prime}\left(-j^{\prime 2}\right)}
$$

Subsequently, the displacement of the midpoint $(\xi=\pi / 2)$ on the equilibrium path Type II is

$$
\Delta u_{j^{\prime} \mathrm{Mid}}=\left(\frac{j^{\prime 2}}{1-j^{\prime 2}} h\right)+\alpha_{j^{\prime}} \sin \frac{j^{\prime} \pi}{2} .
$$

Figure 13 shows this type of equilibrium path with solid gray curves. At the equilibrium state Type III, $\alpha_{4 j} \neq 0$ for some values of $j$, which indicates that the displacement field consists of all mode shapes existing in the Type I solution and an additional asymmetric mode shape $\sin 2 j \xi$. For this type of equilibrium 
path, Eqs. (63)-(65) are applicable when $j^{\prime}$ is replaced by $4 j$ for $j=1,2, \ldots$. In Figure 13, dashed gray lines denote the equilibrium state of Type III.

\subsubsection{Critical points}

The equilibrium path passing through the initial unloaded estate (the primary path) has no critical point. Nevertheless, the secondary equilibrium path, which is a part of the Type I solution, includes limit and bifurcation points. Similarly to the previous sections, it can be proven that all critical points belong to the equilibrium path Type I. Consequently, If $p \neq-n^{2}$ for $n=1,2, \ldots$, the critical condition (34) for limit points is written as

$$
1+\frac{h^{2}}{2(1+p)^{3}}+\frac{\lambda_{\mathrm{Cr}}^{2}}{2} \kappa_{4,3}^{\prime \prime}(p)=0 .
$$

From Eqs. (60) and (66), the value of $\lambda_{\mathrm{Cr}}$ is obtained as a function of $h$ and $\alpha \Delta T_{0}$. Figure 15 displays the locus of limit points over the parameter set $\left\{\left(h, \alpha \Delta T_{0}\right) \in \mathbb{R}^{2} \mid 0<h<10,-10<\alpha \Delta T_{0}<10\right\}$.

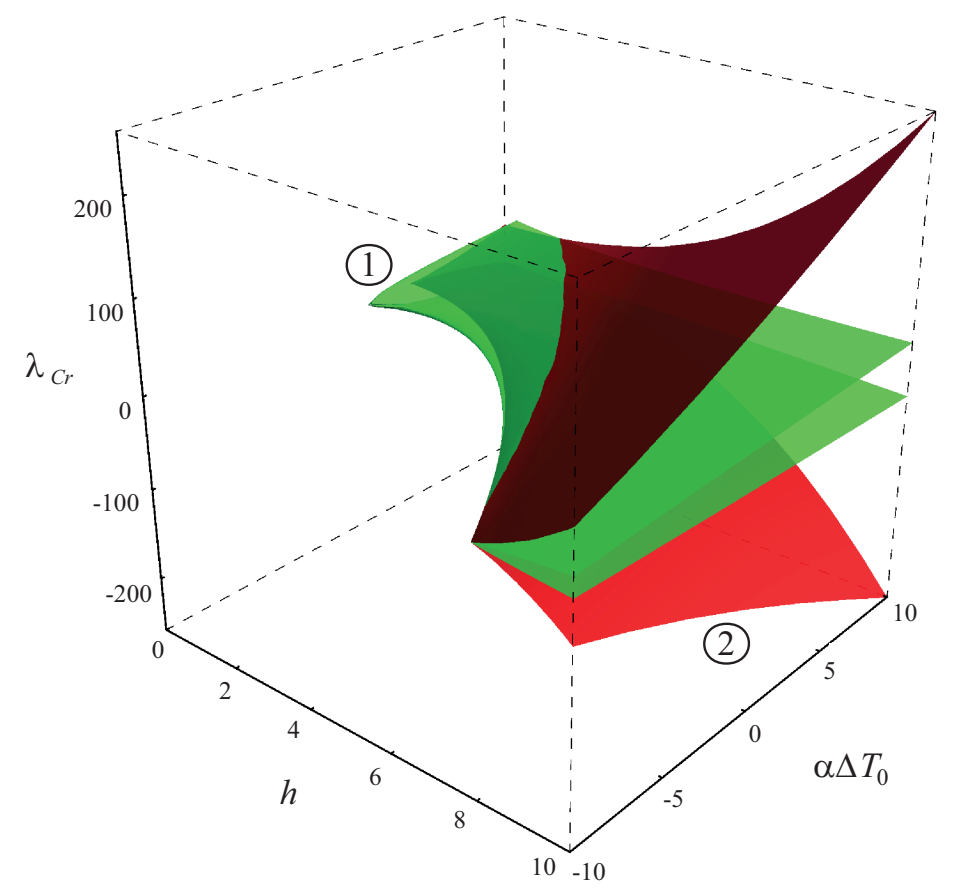

Figure 15: The locus of limit points in the space of $\left(h, \alpha \Delta T_{0}, \lambda_{\mathrm{Cr}}\right)$ 
By projecting the surfaces on the plane of $h$ and $\alpha \Delta T_{0}$, one can isolate areas within this 2-parameter space that correspond to geometries and initial temperature values leading to different number of limit points on the equilibrium path. Due to symmetry (identical behaviour for positive and negative load parameter), $\lambda_{\mathrm{Cr}}=0$ at the boundaries. As a result, by making $\lambda=0$ in Eqs. (60) and (66) and eliminating $p$, the first boundary $B_{1}$ is obtained:

$$
B_{1}=\left\{\left(h, \alpha \Delta T_{0}\right) \in \mathbb{R}^{2} \mid \alpha \Delta T_{0}=1-\frac{h^{2}}{4}+\frac{3 h^{2 / 3}}{2^{4 / 3}}\right\}
$$

Furthermore, $p=-(4 j-2)^{2}$ at the boundaries $B_{j+1}$ for $j=1,2, \ldots$. By substituting this value and $\lambda_{\mathrm{Cr}}=0$ into Eq. (60), the boundaries $B_{j+1}$ are obtained:

$B_{j+1}=\left\{\left(h, \alpha \Delta T_{0}\right) \in \mathbb{R}^{2} \mid \alpha \Delta T_{0}=(4 j-2)^{2}+\left(\frac{1}{4\left(1-(4 j-2)^{2}\right)^{2}}-\frac{1}{4}\right) h^{2}\right\}, j=1,2, \ldots$

Figure 16 shows these boundaries for the interval $\left\{\left(h, \alpha \Delta T_{0}\right) \in \mathbb{R}^{2} \mid 0<h<\right.$ 10, $\left.-10<\alpha \Delta T_{0}<10\right\}$. For the values of $h$ and $\alpha \Delta T_{0}$ within the areas 0,1 and 2, there are zero, two and four limit points in the extra equilibrium path, respectively. The equilibrium paths of the points (a)-(d) are shown in Figure 13. To find the displacement field at critical points for specific values of $h$ and $\alpha \Delta T_{0}$, we first calculate $p$ from Eq. (60). Then, $h, p$ and $\lambda_{\text {Cr }}$ obtained from (66) are substituted into Eq. (61).

Since there are two types of bifurcated paths (Types II and III), two classes of bifurcation points can be observed. Eq. (69) presents the constraints satisfied at bifurcation points:

$$
\left\{\begin{array}{l}
\alpha_{2 i+1}=0 \quad, i=1,2, \ldots \\
\alpha_{4 i}=0 \quad, i=1,2, \ldots \\
p=-\left(j^{\prime}\right)^{2}
\end{array}\right.
$$

where, $j^{\prime}$ is equal to $2 j+1$ in Type II bifurcations and $4 j$ in Type III bifurcations for some $j$. By substituting the value of $p$ into Eq. (60), the locus of bifurcation 


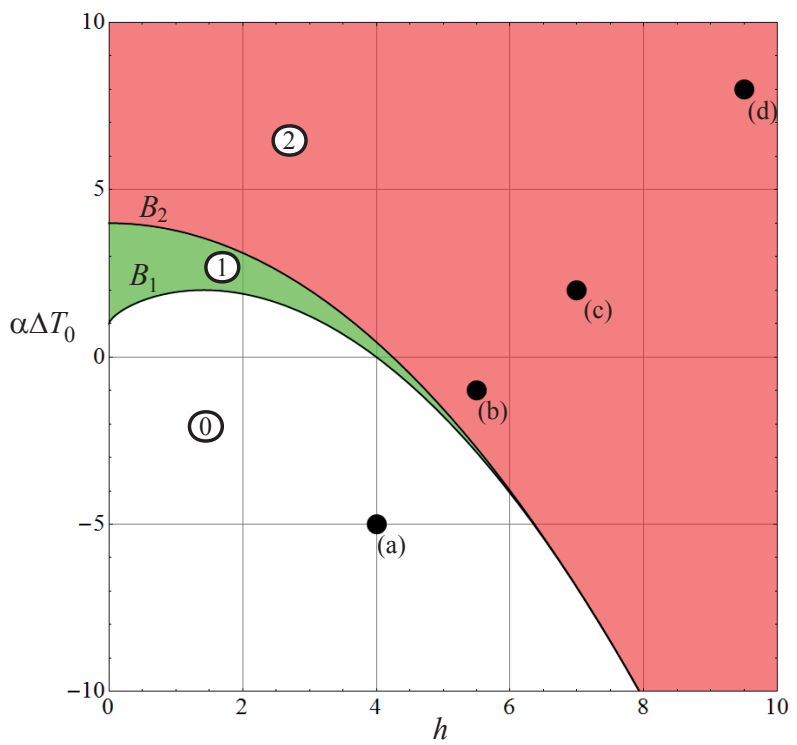

Figure 16: Areas with different number of limit points: zero (area 0), two (area 1) and four (area 2) limit points

points in the space of $\left(h, \alpha \Delta T_{0}, \lambda_{\mathrm{Cr}}\right)$ is obtained:

$$
C_{j^{\prime}}=\alpha \Delta T_{0}+\frac{h^{2}}{4}-j^{\prime 2}-\frac{h^{2}}{4\left(1-j^{\prime 2}\right)^{2}}-\frac{\lambda_{\mathrm{Cr}}^{2}}{4} \kappa_{4,2}^{\prime \prime}\left(-j^{\prime 2}\right)=0
$$

In Figure 17, surfaces 1, 2 and 3 respectively represents $C_{3}, C_{5}$ and $C_{4}$ for the interval $\left\{\left(h, \alpha \Delta T_{0}\right) \in \mathbb{R}^{2} \mid 0<h<10,-10<\alpha \Delta T_{0}<10\right\}$.

The boundaries separating the areas with different number of bifurcation points are the projection of the intersections of surfaces $C_{j^{\prime}}=0$ on the plane of $h$ and $\alpha \Delta T_{0}$. Since the function $C_{j^{\prime}}$ is continuous and differentiable, the constraint $\partial C_{j^{\prime}} / \partial \lambda_{\mathrm{Cr}}=0$ should be satisfied at the boundaries, which gives $\lambda_{\mathrm{Cr}}=0$. From Eq. (70), the boundaries corresponding to $C_{j^{\prime}}=0$ are

$$
B_{j^{\prime}}^{\prime}=\left\{\left(h, \alpha \Delta T_{0}\right) \in \mathbb{R}^{2} \mid \alpha \Delta T_{0}=j^{\prime 2}+\left(\frac{1}{4\left(1-j^{\prime 2}\right)^{2}}-\frac{1}{4}\right) h^{2}\right\} .
$$

Figure 18 shows these boundaries for the interval $\left\{\left(h, \alpha \Delta T_{0}\right) \in \mathbb{R}^{2} \mid 0<h<\right.$ $\left.10,-10<\alpha \Delta T_{0}<10\right\}$. The solid and dashed lines distinguish the number of bifurcation points on the equilibrium paths of Type II and III, respectively.

For the values of $h$ and $\alpha \Delta T_{0}$ within the area (1) (between $B_{3}^{\prime}$ and $B_{4}^{\prime}$ ), 


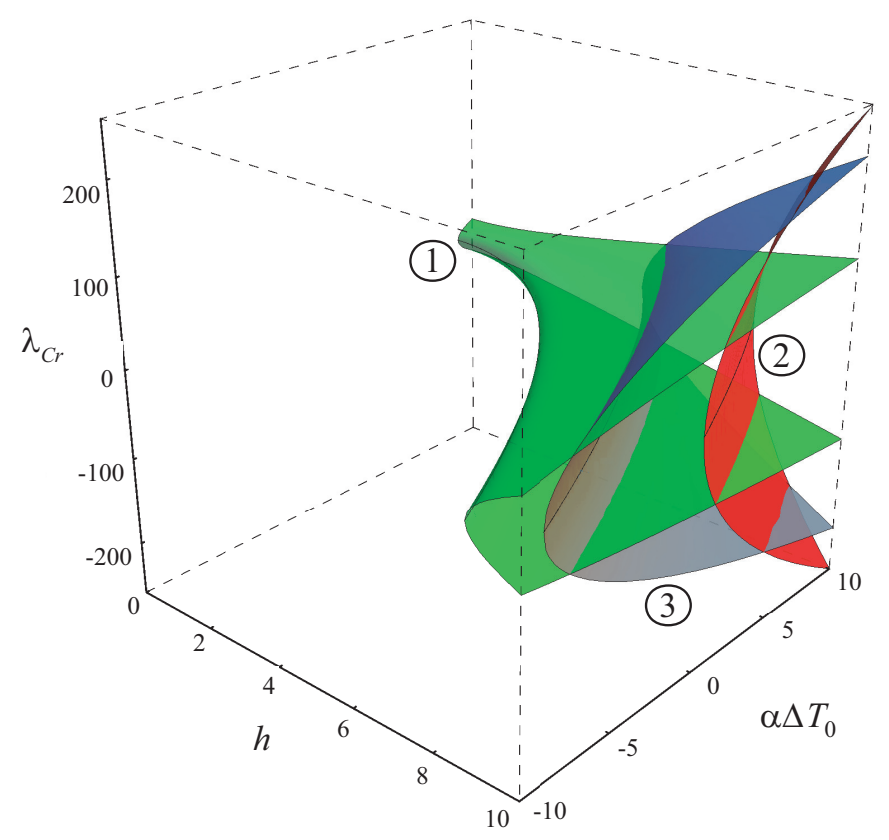

Figure 17: The locus of bifurcation points in the space of $\left(h, \alpha \Delta T_{0}, \lambda_{\mathrm{Cr}}\right)$



Figure 18: Areas with different number of bifurcation points: zero (area 0), two (area 1), four (area 2) and six (area 3) bifurcation points 
there are two bifurcation points relative to Type II, while in the area (3), two additional bifurcation points corresponding to Type III are observed in the equilibrium path. Six bifurcation points exist on the equilibrium path in the area (2).

\section{Conclusions}

In this paper, an analytical method is introduced to identify the complex behaviour of a half-sine pinned shallow arch under static loading in a thermal environment. The governing equations are written in dimensionless form, and the displacement field is decomposed into a Fourier series. The exact solution is formulated based on two independent parameters (the non-dimensionalized rise of the arch and the initial temperature) for arbitrary vertical loadings. The displacement field, equilibrium paths, locus of critical points and natural frequencies are obtained. As examples, the exact solutions of the half-sine shallow arch under concentrated, uniform and asymmetrical uniform loadings are obtained. The accuracy of the proposed method is shown through comparisons with finite element analyses.

The critical states of the arch are also identified. The locus of limit and bifurcation points and the magnitude of load-bearing capacity are derived. Here, it is proven that on the bifurcated path, critical points exist only at its bifurcation points. Furthermore, the boundaries which separate domains with different number of critical points along the equilibrium path are obtained. In addition, the geometry and initial temperature that cause the coincidence of limit and bifurcation points are determined.

The results obtained with the proposed procedure show that elastic shallow arches under concentrated loading at the midpoint and under uniformly distributed loading have similar behaviour, while arches under asymmetrical uniform loading follow a significantly different pattern. For this type of loading, there are equilibrium paths that are not passing through the initial unloaded equilibrium state and do not intersect the primary path but are nevertheless a 
subset of the equilibrium manifold. These paths also contain limit and bifurcation points.

\section{References}

[1] A. B. Pippard, The elastic arch and its modes of instability, European Journal of Physics 11 (1990) 359-365.

[2] J. S. Chen, S. Y. Hung, Exact snapping loads of a buckled beam under a midpoint force, Applied Mathematical Modelling 36 (2012) 1776-1782.

[3] F. Yang, R. Sedaghati, E. Esmailzadeh, Free in-plane vibration of general curved beams using finite element method, Journal of Sound and Vibration 318 (4-5) (2008) 850-867.

[4] Y. Chandra, I. Stanciulescu, T. Eason, M. Spottswood, Numerical pathologies in snap-through simulations, Engineering Structures 34 (2012) 495504.

[5] I. Stanciulescu, T. Mitchell, Y. Chandra, T. Eason, M. Spottswood, A lower bound on snap-through instability of curved beams under thermomechanical loads, International Journal of Non-Linear Mechanics 47 (2012) $561-575$.

[6] J. X. Xu, H. Huang, P. Z. Zhang, J. Q. Zhou, Dynamic stability of shallow arch with elastic supportsapplication in the dynamic stability analysis of inner winding of transformer during short circuit, International Journal of Non-Linear Mechanics 37 (4) (2002) 909-920.

[7] N. J. Mallon, R. H. B. Fey, H. Nijmeijer, G. Q. Zhang, Dynamic buckling of a shallow arch under shock loading considering the effects of the arch shape, International Journal of Non-Linear Mechanics 41 (9) (2006) 1057-1067.

[8] Y. L. Pi, M. A. Bradford, Dynamic buckling of shallow pin-ended arches under a sudden central concentrated load, Journal of Sound and Vibration 317 (3-5) (2008) 898-917. 
[9] G. J. Simitses, An Introduction to the Elastic Stability of Structures, Prentice Hall, Eaglewood Cliffs, New Jersey, 1976.

[10] G. J. Simitses, Dynamic Stability of Suddenly Loaded Structures, SpringerVerlag, New York, 1990.

[11] P. Patricio, M. Adda-Bedia, M. Ben Amar, An elastica problem: instabilities of an elastic arch, Physica D: Nonlinear Phenomena 124 (1-3) (1998) 285-295.

[12] J. S. Chen, C. Y. Liao, Experiment and analysis on the free dynamics of a shallow arch after an impact load at the end, ASME Journal of Applied Mechanics 72 (1) (2005) 54-61.

[13] J. S. Chen, C. H. Yang, Experiment and theory on the nonlinear vibration of a shallow arch under harmonic excitation at the end, ASME Journal of Applied Mechanics 74 (2007) 1061-1070.

[14] J. S. Chen, W. C. Ro, Dynamic response of a shallow arch under end moments, Journal of Sound and Vibration 326 (1) (2009) 321-331.

[15] Y. L. Pi, M. A. Bradford, F. Tin-Loi, Nonlinear analysis and buckling of elastically supported circular shallow arches, International Journal of Solids and Structures 44 (7-8) (2007) 2401-2425.

[16] J. Moon, K. Y. Yoon, T. H. Lee, H. E. Lee, In-plane elastic buckling of pin-ended shallow parabolic arches, Engineering Structures 29 (10) (2007) 2611-2617.

[17] J. S. Chen, W. C. Ro, J. S. Lin, Exact static and dynamic critical loads of a sinusoidal arch under a point force at the midpoint, International Journal of Non-Linear Mechanics 44 (2009) 66-70.

[18] J. S. Chen, J. S. Lin, Dynamic snap-through of a shallow arch under a moving point load, ASME Journal of Vibration and Acoustics 126 (2004) $514-519$. 
[19] J. S. Chen, Y. T. Li, Effects of elastic foundation on the snap-through buckling of a shallow arch under a moving point load, International Journal of Solids and Structures 43 (14) (2006) 4220-4237.

[20] Y. L. Pi, M. A. Bradford, F. Tin-Loi, Non-linear in-plane buckling of rotationally restrained shallow arches under a central concentrated load, International Journal of Non-Linear Mechanics 43 (1) (2008) 1-17.

[21] R. H. Plaut, E. R. Johnson, The effects of initial thrust and elastic foundation on the vibration frequencies of a shallow arch, Journal of Sound and Vibration 78 (4) (1981) 565-571.

[22] J. S. Chen, J. S. Lin, Exact critical loads for a pinned half-sine arch under end couples, ASME Journal of Applied Mechanics 72 (1) (2005) 147-148.

[23] R. H. Plaut, Snap-through of shallow elastic arches under end moments, ASME Journal of Applied Mechanics 76 (2009) 014504.

[24] H. Matsunaga, In-plane vibration and stability of shallow circular arches subjected to axial forces, International Journal of Solids and Structures 33 (4) (1996) 469-482.

[25] S. Y. Hung, J. S. Chen, Snapping of a buckled beam on elastic foundation under a midpoint force, European Journal of Mechanics-A/Solids 31 (1) (2012) 90-100.

[26] J. S. Chen, M. R. Yang, Vibration and stability of a shallow arch under a moving mass-dashpot-spring system, ASME Journal of Vibration and Acoustics 129 (2007) 66-72.

[27] M. A. Crisfield, An arc-length method including line searches and accelerations, International Journal for Numerical Methods in Engineering 19 (9) (1983) 1269-1289.

[28] M. A. Crisfield, Non-linear Finite Element Analysis of Solids and Structures, Volume 1: Essentials, J. Wiley and Sons, Chichester, 1991. 


\section{Appendix A. Functions $\kappa_{M, N}, \kappa_{M, N}^{\prime}$ and $\kappa_{M, N}^{\prime \prime}$}

Here, the functions $\kappa_{M, N}(p), \kappa_{M, N}^{\prime}(p)$ and $\kappa_{M, N}^{\prime \prime}(p)$ are defined, and their magnitudes for some applicable values of $M$ and $N$ are given:

$$
\begin{aligned}
& \kappa_{M, N}(p)=\sum_{i=1}^{\infty} \frac{1}{(2 i+1)^{M}\left((2 i+1)^{2}+p\right)^{N}} \\
& \kappa_{2,1}(p)=\frac{1}{8}\left(-\frac{8}{1+p}+\frac{\pi^{2}}{p}-\frac{2 \pi \tanh \left(\frac{\sqrt{p} \pi}{2}\right)}{p^{3 / 2}}\right) \\
& \kappa_{2,2}(p)= \frac{1}{16 p^{5 / 2}(1+p)^{2}}\left(2 \sqrt{p}\left(-8 p^{2}+(1+p)^{2} \pi^{2}\right)\right. \\
&\left.+(1+p)^{2} \pi \operatorname{sech}\left(\frac{\sqrt{p} \pi}{2}\right)^{2}(\sqrt{p} \pi-3 \sinh (\sqrt{p} \pi))\right) \\
& \kappa_{2,3}(p)= \frac{1}{64 p^{7 / 2}(1+p)^{3}}\left(8 \sqrt{p}\left(-8 p^{3}+(1+p)^{3} \pi^{2}\right)\right. \\
&+(1+p)^{3} \pi\left(-30 \tanh \left(\frac{\sqrt{p} \pi}{2}\right)\right. \\
&\left.\left.+\operatorname{sech}\left(\frac{\sqrt{p} \pi}{2}\right)^{2}\left(7 \sqrt{p} \pi+p \pi^{2} \tanh \left(\frac{\sqrt{p} \pi}{2}\right)\right)\right)\right) \\
&\left.+p(1+p)^{3} \pi^{4}\right)+3(1+p)^{3} \pi\left(70 \tanh \left(\frac{\sqrt{p} \pi}{2}\right)\right. \\
& \kappa_{4,3}(p)= \frac{1}{192 p^{9 / 2}(1+p)^{3}}\left(2 \sqrt { p } \left(-96 p^{4}-36(1+p)^{3} \pi^{2}\right.\right. \\
&\left.+6(1+p)^{2} \pi \operatorname{sech}\left(\frac{\sqrt{p} \pi}{2}\right)^{2}(-\sqrt{p} \pi+5 \sinh (\sqrt{p} \pi))\right) \\
&
\end{aligned}
$$




$$
\begin{gathered}
\kappa_{M, N}^{\prime}(p)=\frac{32 p^{2}+8(1+p) \pi-p(1+p) \pi^{3}-8(1+p) \pi \operatorname{sech}\left(\frac{\sqrt{p} \pi}{2}\right)}{32 p^{2}(1+p)} \\
\kappa_{4,2}^{\prime \prime}(p)=\frac{(-1)^{i+1}}{(2 i+1)^{M}\left((2 i+1)^{2}+p\right)^{N}} \\
\kappa_{4,3}^{\prime \prime}(p)=\frac{1}{384 p^{9 / 2}} \pi\left(\sqrt{p} \pi\left(-144+p \pi^{2}-66 \operatorname{sech}\left(\frac{\sqrt{p} \pi}{4}\right)^{2}\right)\right. \\
\left.-3\left(-280+p \pi^{2} \operatorname{sech}\left(\frac{\sqrt{p} \pi}{4}\right)^{2}\right) \tanh \left(\frac{\sqrt{p} \pi}{4}\right)\right)
\end{gathered}
$$

